ACCEPTED MANUSCRIPT

\title{
Temperature effect on the magnetic oscillations in 2D materials
}

To cite this article before publication: Federico Nahuel Escudero et al $2019 \mathrm{~J}$. Phys.: Condens. Matter in press https://doi.org/10.1088/1361$\underline{648 \mathrm{X} / \mathrm{ab} 14 \mathrm{~b} 6}$

\section{Manuscript version: Accepted Manuscript}

Accepted Manuscript is "the version of the article accepted for publication including all changes made as a result of the peer review process, and which may also include the addition to the article by IOP Publishing of a header, an article ID, a cover sheet and/or an 'Accepted Manuscript' watermark, but excluding any other editing, typesetting or other changes made by IOP Publishing and/or its licensors"

This Accepted Manuscript is ( 2019 IOP Publishing Ltd.

During the embargo period (the 12 month period from the publication of the Version of Record of this article), the Accepted Manuscript is fully protected by copyright and cannot be reused or reposted elsewhere.

As the Version of Record of this article is going to be / has been published on a subscription basis, this Accepted Manuscript is available for reuse under a CC BY-NC-ND 3.0 licence after the 12 month embargo period.

After the embargo period, everyone is permitted to use copy and redistribute this article for non-commercial purposes only, provided that they adhere to all the terms of the licence https://creativecommons.org/licences/by-nc-nd/3.0

Although reasonable endeavours have been taken to obtain all necessary permissions from third parties to include their copyrighted content within this article, their full citation and copyright line may not be present in this Accepted Manuscript version. Before using any content from this article, please refer to the Version of Record on IOPscience once published for full citation and copyright details, as permissions will likely be required. All third party content is fully copyright protected, unless specifically stated otherwise in the figure caption in the Version of Record.

View the article online for updates and enhancements. 


\title{
Temperature effect on the magnetic oscillations in $2 \mathrm{D}$ materials
}

\author{
F Escudero, J S Ardenghi, P Jasen
}

IFISUR, Departamento de Física (UNS-CONICET), Av. Alem 1253, B8000CPB

Bahía Blanca, Argentina

Instituto de Física del Sur (IFISUR, UNS-CONICET)

Av. Alem 1253, B8000CPB Bahía Blanca, Argentina

E-mail: federico.escudero@uns.edu.ar

\begin{abstract}
We study the magnetic oscillations (MO) in 2D materials with a buckled honeycomb lattice, considering a perpendicular electric and magnetic field. At zero temperature the MO consist of the sum of four sawtooth oscillations, with two unique frequencies and phases. The values of these frequencies depend on the Fermi energy and electric field, which in turn determine the condition for a beating phenomenon in the MO. We analyse the temperature effect in the MO by considering its local corrections over each magnetization peak, given by Fermi-Dirac like functions. We show that the width of these functions is related to the minimum temperature necessary to observe the spin and valley properties in the MO. In particular, we find that in order to observe the spin splitting, the width must belower than the MO phase difference. Likewise, in order to observe valley mixing effects, the width must be lower than the MO period. We also show that at high temperatures, all the maxima and minima in the MO are shift to a constant value, in which case we obtain a simple expression for the MO and its envelope. The results obtained show unique features in the MO in 2D materials, given by the interplay between the valley and spin.
\end{abstract}

\section{Introduction}

Since the experimental realization of graphene in 2004 [1, 2, 3], many similar planar systems have been studied [4, 5, 6]. Among them are silicene [7, 8, 9, 10], germanene [11, 12], stanene [13, 14] and phosphorene [15, 16]. These materials have a 2D hexagonal lattice, made of two buckled subtallices $\mathrm{A}$ and $\mathrm{B}$. They are best described with a tight binding (TB) model, which leads to an effective Dirac-like Hamiltonian in the low energy approximation [17, 18]. Thus these materials are also referred as Dirac crystals. Despite their similarities, there are important features that distinguish one material from another. One is the spin-orbit interaction (SOI), which is very small in graphene (about $10^{-3} \mathrm{meV}[19]$ ), but relatively large in other materials (for instance, it is $0.1 \mathrm{eV}$ in stanene [17]), which makes them a topological insulator [20, 21, 22, 23]. Moreover, a strong SOI would make possible the observation of the quantum spin Hall effect [24, 25, 26, 27, 28, 29]. Another characteristic is the buckle height, which defines 


\section{Temperature effect on the magnetic oscillations in $2 D$ materials}

the layer separation between the two sublattices. In graphene this buckle height is zero [19], but it is not zero in the other Dirac crystals [6]. Hence, applying a perpendicular electric field between the two sublattices causes a potential difference, which splits the energy bands and can be used to tune the bandgap [30, 31, 32, 33, 34, 35, 36].

The magnetic properties of the Dirac crystals have been investigated in recent works [37, 38, 39, 40, 41]. Unlike in conventional materials, the magnetization in these systems has unique features [42, 43, 44, 45, 46, 47]. A particular interesting phenomenon are the magnetic oscillations (MO), the so called de Haas van Alphen effect [48, produced by the discrete energy levels that appear when a magnetic field is applied. At zero temperature the MO are sawtooth [40], with the peaks been caused by the change in the last occupied energy level [49. Therefore the MO depend strongly on the system energy levels. In the Dirac crystals, at low energies the dispersion relation is relativistic [19], which causes the Landau levels (LL) to be not equidistant [50, 51]. These anomalous LL can be modify by external parallel and perpendicular electric fields [52, 50]. For instance, the MO in graphene can be modulated by an in-plane electric field [53], which leads to unique features not seen in the conventional 2D electron gas. In the Dirac crystals, a perpendicular electric field alters the LL due to the buckled height and strong SOI [47, which can have an appreciable effect in the MO.

At non zero temperature the MO are broadened as a result of the Fermi-Dirac distribution. In classical metals this is described by the Lifshitz-Kosevich (LK) formula [48], which incorporates the temperature effect as a reduction factor. This formula has been extended to the case of Dirac crystals [37, where the difference only lies in the form of the reduction factor. Another approach, recently developed in graphene [54], considers the temperature effect by local corrections over each MO peak. This is particular useful at very low temperatures, where the MO are modified only around the peaks location at $T=0$. Nevertheless, there is no detailed analysis about how the fine structure of the $\mathrm{MO}$ in $2 \mathrm{D}$ buckled materials is progressively lost as the temperature increases. This is particular relevant from an experimental point of view, since there is always a limit to how low the temperature can be.

Motivated by this we analysed the MO in a general pristine Dirac crystal, in the presence of a perpendicular electric and magnetic field, taking into account the Zeeman effect. We have organized this work as follow: in section 2 we describe the MO at zero temperature, showing that it consist of two unique frequencies and phases. Then we study the dependence of these frequencies with the electric field, and the condition for a beating phenomenon. In section 3 we study how the temperature broadens the MO and affectsits observation. We estimate the minimum temperature required to observe the valley and spin properties. At high temperature we also obtain a simple approximation for the MO and its envelope. Finally, our conclusions follow in section 4 . 


\section{MO at zero temperature}

We shall study the MO in a general 2D system with a buckled honeycomb structure. Examples of these materials are silicene, germanene, stanene and phosphorene. We will consider energies close to the Fermi level, in which case one can apply the long wavelength approximation [19, 17]. Then, in the presence of a perpendicular electric field $E_{z}$, these materials are described by a Dirac Hamiltonian of the form [18]

$$
H=v_{\mathrm{F}}\left(\eta p_{x} \sigma_{x}+p_{y} \sigma_{y}\right)+\kappa_{s}^{\eta} \sigma_{z},
$$

where $v_{\mathrm{F}}$ is the Fermi velocity, $\boldsymbol{\sigma}$ are the Pauli matrices and $\kappa_{s}^{\eta}=\eta s \lambda_{\mathrm{SO}}-e l E_{z}$, with $\lambda_{\text {SO }}$ the spin-orbit coupling interaction (SOI) and $l$ the buckle height. The indices $\eta$ and $s$ are the valley and spin indices, with values $1(-1)$ for the $K\left(K^{\prime}\right)$ valley and spin up (down). The particular values of $v_{\mathrm{F}}, \lambda_{\mathrm{SO}}$ and $l$ depend on the $2 \mathrm{D}$ material. It is worth noting that graphene can be considered a special case, with $l=0$ and $\lambda_{\mathrm{SO}} \simeq 0$. In the presence of a perpendicular magnetic field $B$, the Hamiltonian given by equation (1) gives the energy levels [47] $\varepsilon_{\zeta, n, \eta, s}=\zeta\left[\left(s \lambda_{\mathrm{SO}}-\eta \mathrm{l} E_{z}\right)^{2}+\alpha^{2} n B\right]^{1 / 2}-s \mu_{\mathrm{B}} B$, where $\zeta= \pm 1$ for the conduction and valence bands, $\alpha=v_{\mathrm{F}} \sqrt{2 \hbar e}, n=0,1,2, \ldots$ for the Landau level (LL) and we took into account the Zeeman term $\mu_{\mathrm{B}} B$. Each energy level has a degeneracy given by $D=\mathcal{A} B / \phi$, where $\mathcal{A}$ is the sheet area and $\phi=h / e$ is the magnetic unit flux [50]. We will take a constant Fermi energy $\mu>0$, so that only the conduction band contributes to the MO. Then the problem becomes analogue to the one already studied in graphene [54], with the inclusion of the term $\kappa_{s}^{\eta}$. In this way, generalizing this approach we get that the MO are given by (see the Appendix A for details)

$$
M=\sum_{i=1,2} \frac{A_{i}}{\pi} \sum_{s \neq \pm 1} \arctan \left\{\cot \left[\pi \omega_{i}\left(\frac{1}{B}+s \Delta_{i}\right)\right]\right\},
$$

where

Therefore the MO at zero temperature consist of four type of peaks, corresponding to the possible combinations of valley and spin. There are two unique frequencies $\omega_{1}$ and $\omega_{2}$, with phases $\Delta_{1}$ and $\Delta_{2}$. This result generalizes the graphene case, and it says that the broken valley degeneracy in buckled $2 \mathrm{D}$ materials is seen in the MO as two oscillations with different frequency [38]. The values of these frequencies and phases depend on the properties of the Dirac crystal, such as the SOI, the buckle height 
and Fermi velocity, as well as the Fermi energy and the perpendicular electric field. Therefore, these parameters define the conditions for which the peaks can occur, for that implies $\omega>0$. In graphene, the buckle height is zero and the SOI negligible, so there is only one frequency $\omega_{g}=\omega_{1}=\omega_{2}=\mu^{2} / \alpha^{2}$ and two peaks with phase difference between them $\Delta_{g}=2 \mu \mu_{\mathrm{B}} \omega_{g} \alpha^{2}$; the condition $\omega_{g}>0$ just implies $\mu>0$. For the other crystals, the condition $\omega>0$ implies $\mu^{2}>\left(\lambda_{\mathrm{SO}} \pm e l E_{z}\right)^{2}$, so we have the regions indicated in figure 1, corresponding to stanene. Depending on the value of the Fermi energy and the electric field, three possibilities can occur: (I) $\omega_{1}>0$ and $\omega_{2}>0$, so all 4 peaks are present; (II) $\omega_{1}>0$ and $\omega_{2}<0$, so only two peaks with frequency $\omega_{1}$ and phase difference $\Delta_{1}$ are present; (III) $\omega_{1}<0$ and $\omega_{2}<0$, so there are no peaks and therefore no MO (the magnetization would be given only by the regular, non-oscillatory contribution). Notice that $\omega_{2}$ always decrease with increasing $E_{z}$, while $\omega_{1}$ increases with $E_{z}$ for $e l E_{z}<\lambda_{\mathrm{SO}}$, it takes its maximum at $e l E_{z}=\lambda_{\mathrm{SO}}$ (where $\omega_{1}=\mu^{2} / \alpha^{2}$ as in graphene), and then decrease with increasing $E_{z}$ for $e l E_{z}>\lambda_{S O}$. It should be noted that the MO given by equation (2) equals the total magnetization only when $\mu>\left|\lambda_{\mathrm{SO}} \pm e l E_{z}\right|$ [37, which implies $\omega_{i}>0$. Thus only when both $\omega_{1}$ and $\omega_{2}$ are present (region $I$ in figure 11), the total magnetization is given by equation (2). In the other regions, one has also to consider the regular and vacuum contributions to the total magnetization.

The relationship between the MO and $\omega>0$ can be better understand by analysing

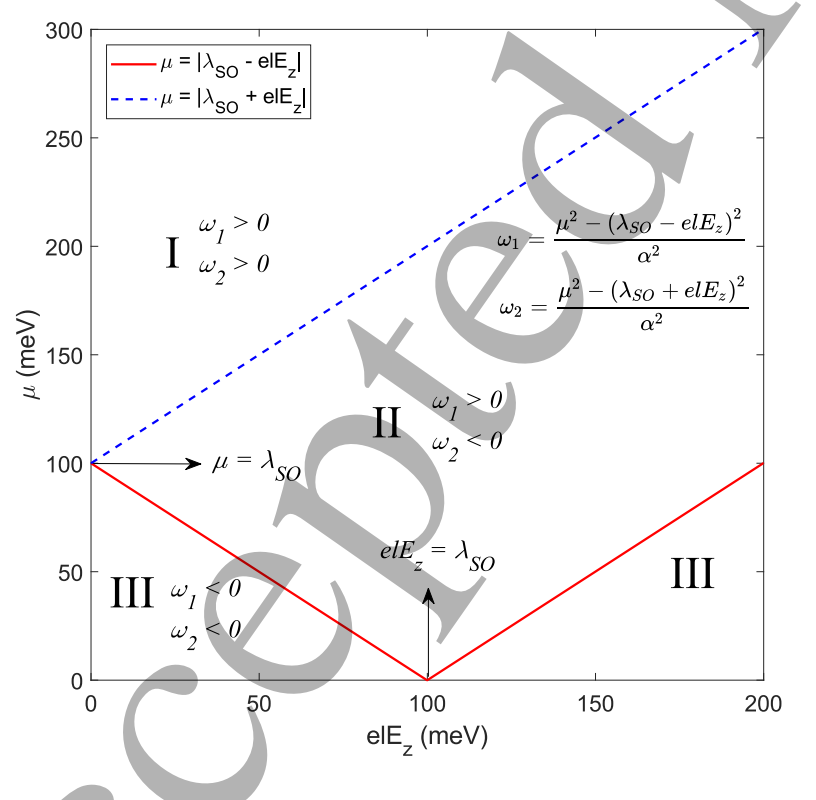

Figure 1. MO frequency spectrum for stanene, as a function of the perpendicular electric field $E_{z}$ and the Fermi energy $\mu$. The presence of $E_{z}$ produces a broken valley degeneracy, which results in two frequencies $\omega_{1}$ and $\omega_{2}$ for the MO, with $\omega_{1}>\omega_{2}$ always. The oscillations occur only if $\omega>0$, which defines the three regions shown: (I) $\omega_{1}>0$ and $\omega_{2}>0$, so both frequencies are present in the MO, (II) $\omega_{1}>0$ and $\omega_{2}<0$, in which case the magnetization oscillates with only one frequency, as in graphene, and (III) $\omega_{1}<0$ and $\omega_{2}<0$, so there is no MO. 
1

2

3

4

5

6

7

8

9

the energy level change that produce the oscillation. First of all, for a constant $\mu$ we have the energy levels $\varepsilon_{i}=\left[\mu^{2}+\alpha^{2}\left(n B-\omega_{i}\right)\right]^{1 / 2}-s \mu_{\mathrm{B}} B$ associated with $\omega_{i}$, given by equation (4). Then $\omega_{i}<0$ implies $\left(\varepsilon_{i}+s \mu_{\mathrm{B}} B\right)^{2}>\mu^{2}+n \alpha^{2} B$, but the occupied energy levels satisfy $\varepsilon_{i}<\mu$. Hence, given that in general $\mu_{\mathrm{B}} B / \mu \ll 1$, for $B>0$ (maintaining the magnetic field direction), we have that $\varepsilon_{i}$ is never occupied if $\omega_{i}<0$, so there is no oscillation associated with a change of $\varepsilon_{i}$. In the particular case (II) in figure 1, we have $\left(\lambda_{S O}-e l E_{z}\right)^{2}<\mu^{2}<\left(\lambda_{S O}+e l E_{z}\right)^{2}$, and the last LL $n$ occupied in $\varepsilon_{1}$ satisfy $\left(\lambda_{S O}-e l E_{z}\right)^{2}+n \alpha^{2} B<\left(\lambda_{S O}+e l E_{z}\right)^{2}<\left(\lambda_{S O}-e l E_{z}\right)^{2}+(n+1) \alpha^{2} B$. Thus, when $\omega_{1}>0$ and $\omega_{2}<0, \varepsilon_{2}$ is not occupied and there are $n=$ Floor $\left[\left(\omega_{1}-\omega_{2}\right) / B\right] \mathrm{LL}$ occupied in $\varepsilon_{1}$. We see that $n$ depends on $B$, and its value is given by the ratio between the frequency difference and the magnetic field. This is expected considering that the magnetization oscillates as a function of $1 / B$.

In the general case, when both frequencies are present, the MO will show an interference pattern, produced by the superposition of $M_{1}$ and $M_{2}$, each one being a sawtooth oscillation. The specific pattern in the MO will, in general, depend on the values of $\omega_{1}$ and $\omega_{2}$. The most interesting situation occurs when $\omega_{1}$ and $\omega_{2}$ are close, in which case the MO show a beating phenomenon. Given that always $\omega_{1}>\omega_{2}$, the beating condition is $\left(\omega_{1}-\omega_{2}\right) / \omega_{1} \ll 1$. In figure 2 it is plotted $\left(\omega_{1}-\omega_{2}\right) / \omega_{1}$ for silicene, at different values of $\mu$, as a function of the perpendicular electric field. We observe that the lower the Fermi energy, the lower the range of electric field for which there is beating. In the practice, a clear beating phenomenon is observed as long as $\left(\omega_{1}-\omega_{2}\right) / \omega_{1} \lesssim 0.1$. When $\left(\omega_{1}-\omega_{2}\right) / \omega_{1} \ll 1$ is not satisfied, there is still an interference in the MO, only
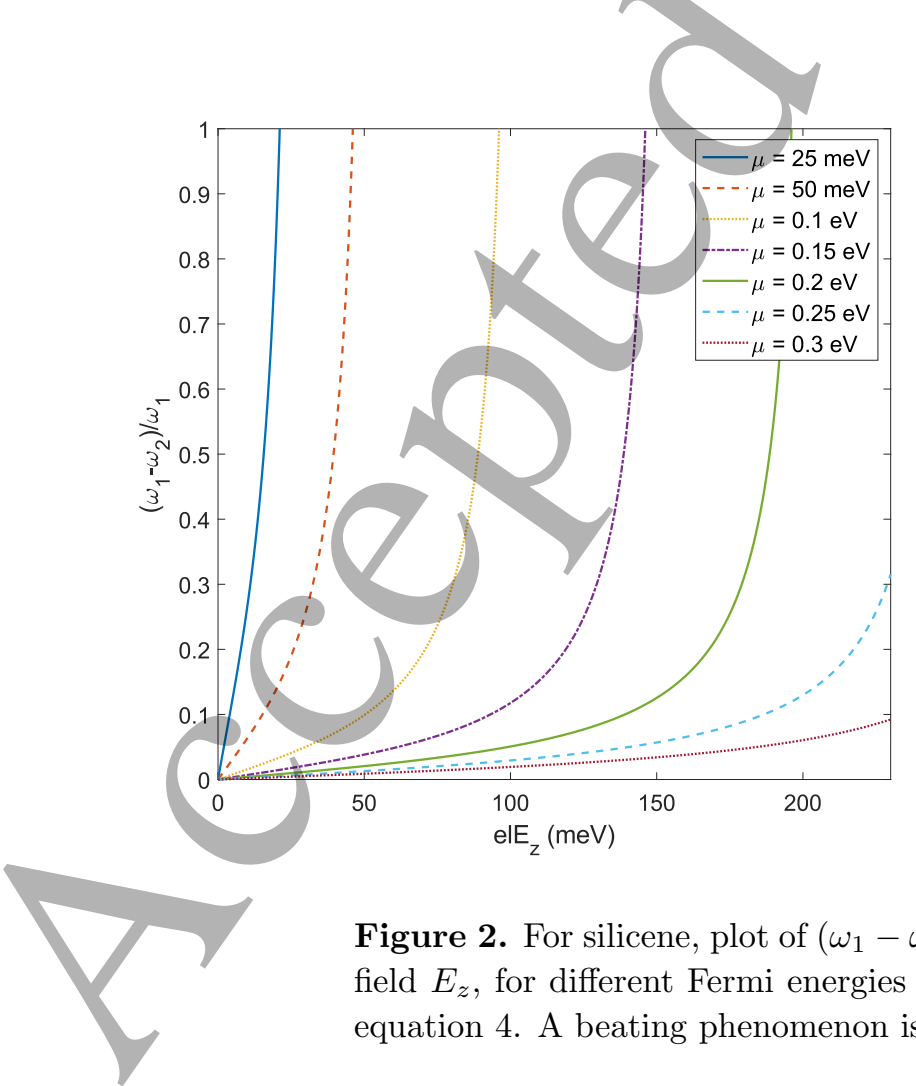

Figure 2. For silicene, plot of $\left(\omega_{1}-\omega_{2}\right) / \omega_{1}$ as a function of the perpendicular electric field $E_{z}$, for different Fermi energies $\mu$. The MO frequencies $\omega_{1}$ and $\omega_{2}$ are given by equation 4. A beating phenomenon is observed only when $\left(\omega_{1}-\omega_{2}\right) / \omega_{1} \ll 1$. 
that it would not be seen as a beating phenomenon. Instead the MO show a more random-like pattern, where the behaviour depends specifically on the particular values of $\omega_{1}$ and $\omega_{2}$. This can be seen in figure 3, where we plotted the MO in silicene for $\mu=0.2 \mathrm{eV}$, at el $E_{z}=70 \mathrm{meV}$ and $e l E_{z}=180 \mathrm{meV}$. This drastic change of behaviour in the MO can be explained by analysing how the energies levels are sorted in each case. When there is beating, $\omega_{1}$ and $\omega_{2}$ are close and so are the respective energy levels $\varepsilon_{i, n}$ (with the same LL $n$ ) that give rise to these frequencies, as discussed above. Thus the change of the last energy level (which produces the MO) follows an ordered pattern that interchanges $\varepsilon_{1, n}$ and $\varepsilon_{2, n}$ as $B$ is changed. On the other hand, when $\omega_{1}$ and $\omega_{2}$ are far apart, such that $\left(\omega_{1}-\omega_{2}\right) / \omega_{1} \ll 1$ is not satisfied, then the energy leyels $\varepsilon_{1, n}$ and $\varepsilon_{2, n}$ are not close and there is no clear pattern in the change of the last energy level. In this region, the sort of the energy levels depends on the value of $E_{z}$ and $B$, leading to a seemingly random pattern in the MO. However, it should be noted that if one analyses the specific order of the energy levels at a particular $E_{z}$, then the MO behaviour can be explained [47.

We shall now analyse in more detail the beating phenomenon in the MO at zero temperature. As we can see in figure 3(a), the beating has a rombo-like pattern,

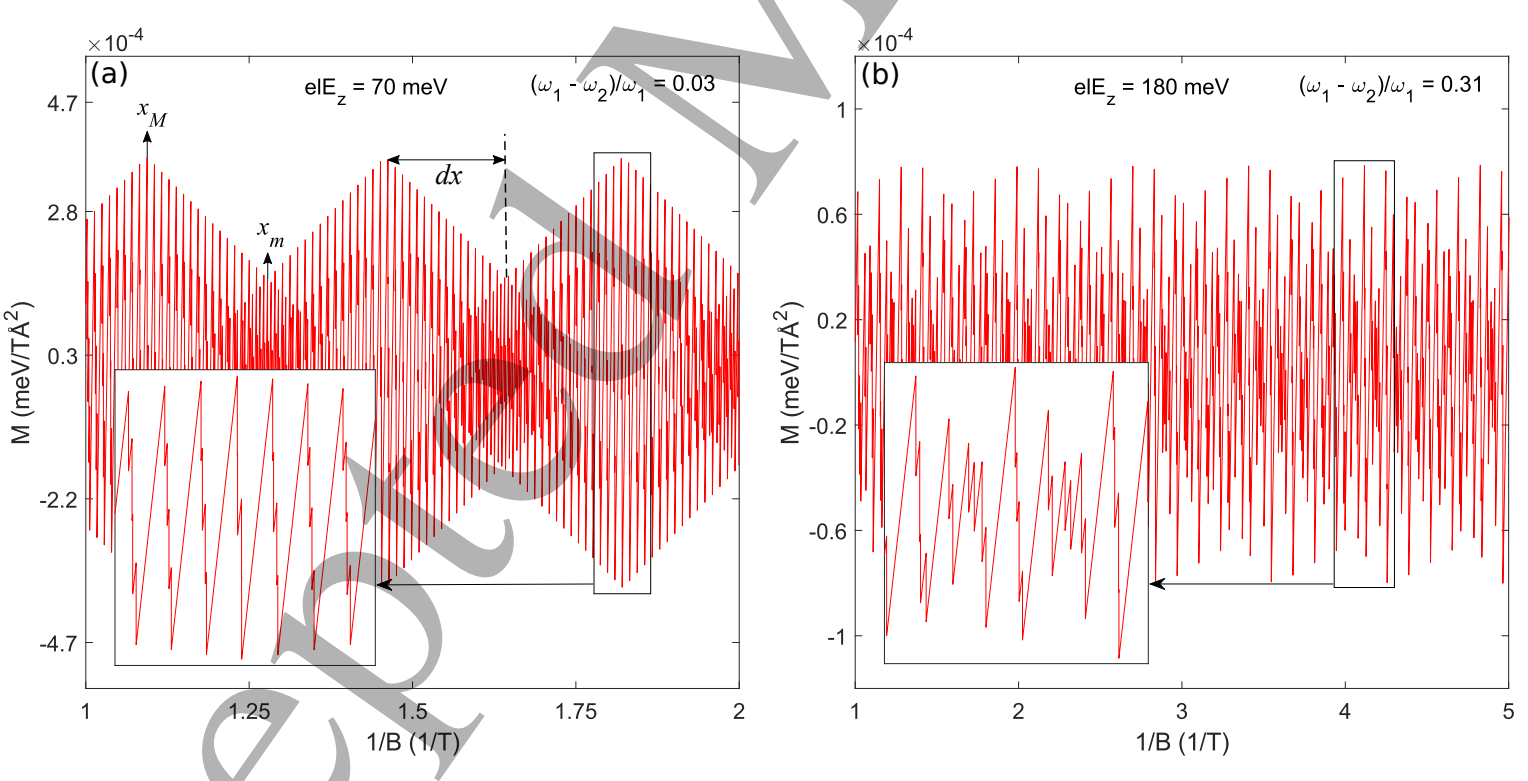

Figure 3. $\mathrm{MO}$ in silicene at zero temperature, with a Fermi energy $\mu=0.2 \mathrm{eV}$, for (a) $\left(\omega_{1}-\omega_{2}\right) / \omega_{1}=0.03$ and (b) $\left(\omega_{1}-\omega_{2}\right) / \omega_{1}=0.31$. In (a) we see a clear beating phenomenon, consistent with the condition $\left(\omega_{1}-\omega_{2}\right) / \omega_{1} \ll 1$. The beating is seen as a rombo-like pattern, produced by the superposition of the sawtooth oscillations with frequencies $\omega_{1}$ and $\omega_{2}$. The beating maxima and minima are $x_{M}=r /\left(\omega_{1}-\omega_{2}\right)$ and $x_{m}=(r+1 / 2) /\left(\omega_{1}-\omega_{2}\right)$, with $r$ an integer, and their distance is $d x=1 / 2\left(\omega_{1}-\omega_{2}\right)=\hbar v_{\mathrm{F}}^{2} / 4 \lambda_{\mathrm{SO}} l E_{z}$. In (b), the beating condition is not satisfied, resulting in a more random-like pattern in the MO. In this situation, the order of the peaks depends strongly not only on the value of $E_{z}$, but also on the magnetic field $B$. 


\section{Temperature effect on the magnetic}

oscillations in $2 D$ materials

caused by both $M_{1}$ and $M_{2}$ being a sawtooth oscillation. If we restrict to few values of magnetic field, one can appreciate the fine structure of the MO, as can be seen in the zoomed area. In this region one explicitly sees the four MO peaks given by equation (2), with their amplitude and phase being determined by the change of spin and valley in the last energy level. The absolute maxima $1 / B_{M} \equiv x_{M}$ in the MO occur when there is constructive interference. From equations (2), (4) and (5), this implies $x_{M}=m_{1} / \omega_{1} \pm \Delta_{1}=m_{2} / \omega_{2} \pm \Delta_{2}$, where $m_{1}$ and $m_{2}$ are integer such that $m_{2}=m_{1}+r$, with $r$ an integer. Given that in general $\omega \Delta \ll 1$, we get that the maxima occur at $x_{M}=r /\left(\omega_{1}-\omega_{2}\right)$. The absolute minima happen between two maxima, so $x_{m}=(r+1 / 2) /\left(\omega_{1}-\omega_{2}\right)$, and the width between maxima and minima is $d x=1 / 2\left(\omega_{1}-\omega_{2}\right)=\hbar v_{\mathrm{F}}^{2} / 4 \lambda_{\mathrm{SO}} l E_{z}$. Therefore, one can obtain information about the material parameters by measuring the width of the rombo-like pattern in the MO. Notice that $\left(\omega_{1}-\omega_{2}\right)$ does not depend on the magnetic field or Fermi energy, but only on the electric field. This is expected because the width depends on the frequency difference of the peaks, which is only produced by the perpendicular electric field.

\section{MO at non zero temperature}

We shall now study the temperature influence in the MO. We will consider low $B$ and $T$, such that we can neglect the effect of lattice vibrations [55, 56]. The temperature effect in the MO can be taken into account in different ways. The most common one is by the Lifshitz-Kosevich (LK) formula [48, in which the damping effects such as the temperature are considered by reductions factors. Thus, in the pristine case (i.e. no impurities), the MO at $T \neq 0$ are $[37]$

$$
M_{T}=\sum_{i=1,2} A_{i} \sum_{s= \pm 1} \sum_{p=1}^{\infty} \frac{R_{T}}{\pi p} \sin \left[2 \pi p \omega_{i}\left(\frac{1}{B}+s \Delta_{i}\right)\right]
$$

where $R_{T}=\lambda p / \sinh (\lambda p)$ with $\lambda=4 \pi^{2} \mu k_{\mathrm{B}} T / \alpha^{2} B$. Another way to express the MO at non zero temperature is by considering the local corrections to each peak due the Fermi-Dirac distribution, as has been done for graphene [54]. Generalizing this result we obtain (see the Appendix B for details)

$$
M_{T}=\sum_{i=1,2} \frac{A_{i}}{\pi} \sum_{s= \pm 1} \arctan \left\{\cot \left[\pi \omega_{i}\left(\frac{1}{B}+s \Delta_{i}\right)+\sum_{n} \pi \mathcal{F}_{i, n}\right]\right\}
$$

where $\mathcal{F}_{i, n}=\left\{1+\exp \left[\beta \mu_{\mathrm{B}}\left(B_{n}-B\right) / B_{n} \Delta_{i}\right]\right\}^{-1}$, with $\beta=1 / k_{\mathrm{B}} T$ and $B_{n}^{-1}=n / \omega_{i}-s \Delta_{i}$ being the MO peaks location at $T=0$. It is instructive to compare these two expressions for the MO at $T \neq 0$, because depending on the situation it may be convenient to use one or the other formula. It is important to note that both expressions give the same MO at $T \neq 0$; they are just two different ways of expressing the same. The series given by equation (6) express the MO as a sum of harmonics, which in some situations could be useful, in particular when one can isolate the contribution from each harmonic. At 
low temperature the summation cannot be exactly solved, although it should be noted that few terms are needed in the infinite sum, since the factors decay rapidly with $p$. At high temperatures, such that $\sinh (\lambda p) \sim \exp (-\lambda p)$, the summation can be solved, leading to a simple expression for the MO. On the other hand, the expression given by equation (7) considers the temperature influence by local corrections around each $\mathrm{MO}$ peak at $T=0$. Indeed, each term inside the summation over $n$ is a Fermi-Dirac like function, which at low temperature is appreciable only around $B_{n}$. This is particular useful to analyse in detail how the increase in the temperature affects the observation of the MO fine structure, such as the spin splitting and valley mixing.

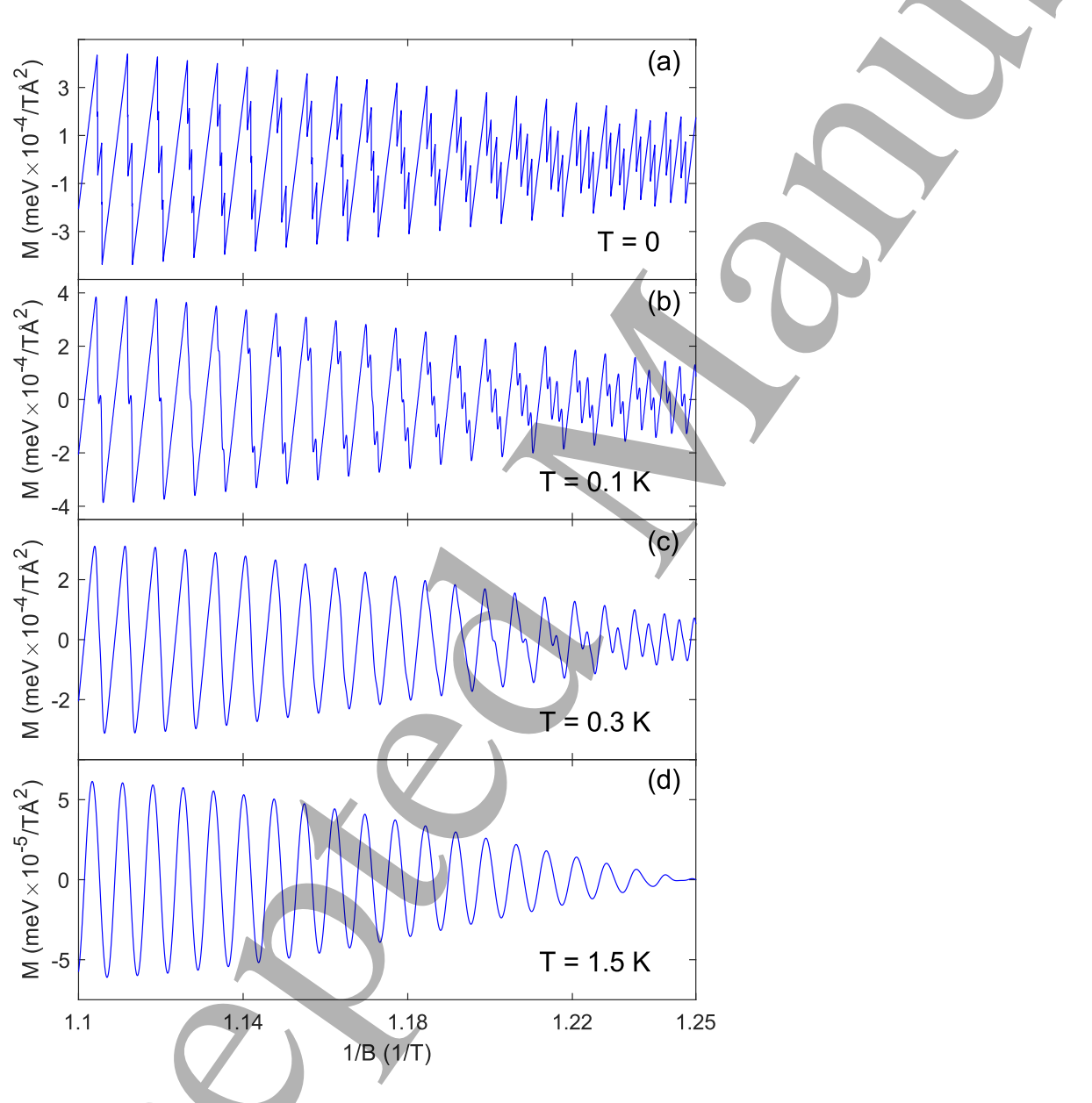

Figure 4. $\mathrm{MO}$ in silicene, for different temperatures, with $\mu=0.25 \mathrm{eV}$ and $e l E_{z}=92$ $\mathrm{meV}$, resulting in a beating phenomenon with $\left(\omega_{1}-\omega_{2}\right) / \omega_{1} \sim 0.03$. All cases shown correspond to the region between the beating maximum at $x_{M}=4 /\left(\omega_{1}-\omega_{2}\right)$ and the minimum at $x_{m}=4.5 /\left(\omega_{1}-\omega_{2}\right)$. In (a) we see the MO at $T=0$, where we can observe the fine structure of the MO, due to the spin splitting (SP) and valley mixing (VM). For $T=0.1 \mathrm{~K}$ in (b), the $\mathrm{MO}$ are now damped, but one can still observe the SP. Increasing further the temperature to $T=0.3 \mathrm{~K}$ in (c), the small peaks due to the SP disappear, although the VM around the beating minimum still can be seen. If we increase further the temperature to $T=1.5 \mathrm{~K}$ in (d), then the VM also disappears in the MO. Thus we say that at this stage the fine structure of the MO is damped due to the temperature. 
In figure 4 we show the MO for different temperatures, in the case of silicene with $\mu=0.25 \mathrm{eV}$ and $\mathrm{el} E_{z}=92 \mathrm{meV}$. This gives $\omega_{1}>0$ and $\omega_{2}>0$, with $\left(\omega_{1}-\omega_{2}\right) \simeq 3.6$ $\mathrm{T}$, so we are plotting the region between the maximum at $x_{M}=4 /\left(\omega_{1}-\omega_{2}\right)$ and the minimum at $x_{m}=4.5 /\left(\omega_{1}-\omega_{2}\right)$. The temperatures considered, for this particular case, are specifically chosen to represent how they affect the observation of the spin splitting (SP) and the valley mixing (VM) behaviour that occurs around the beating minimum. Thus, starting from the $T=0$ case in figure 4(a), we clearly observe the fine structure of the MO, due to the spin and valley. As we increase the temperature, all the peaks start to broaden, and depending on $T$, some peaks would no longer be observed. First of all, in figure $4(\mathrm{~b})$, we see that at $T=0.1 \mathrm{~K}$, the peaks are now broaden, but nevertheless one could still appreciate the SP in the MO, which is seen as the small bumps between the bigger peaks [49]. But if we continue increasing the temperature, we get to the situation shown in figure $4(\mathrm{c})$, where for $T=0.3 \mathrm{~K}$ the $\mathrm{MO}$ are broaden such that the $\mathrm{SP}$ cannot be observed any more. However, we still see the VM behaviour in the MO when we are at the minimum region $(1 / B$ around $1.25 \mathrm{~T})$, due to the broken valley degeneracy. Increasing the temperature further, we eventually get to the state shown in figure $4(\mathrm{~d})$, where the VM also disappears. This last state is maintained when the temperature continues to increase, where the MO are more damped but the form does not change, corresponding to a pure beating phenomenon. We shall now study in detail the situations considered, namely how we can estimate in general the temperature at which the SP and VM would no longer be observed. As discussed above, in this low temperature situation it will be more useful to use equation (7). In order to do that we will first analyse how the last term in equation (7) alters the observation of each $\mathrm{MO}$ peak at non zero temperature.

\subsection{Temperature effect over each MO peak}

We will study the temperature effect over each MO peak, which will be useful in the subsequent analysis. Thus we consider, in general, a unique MO peak at a given $B_{0}$ and we omit the effect of others peaks close to $B_{0}$ (either due to spin splitting or valley mixing). Then, from equation 7 the magnetization is (we will take $s=1$, but the analysis is valid for any spin and valley)

$$
M_{T}=\frac{A}{\pi} \arctan \left\{\cot \left[\pi \omega\left(\frac{1}{B}+\Delta\right)+\pi \mathcal{F}_{0}\right]\right\}
$$

with $\mathcal{F}_{0}=\left\{1+\exp \left[\beta \mu_{\mathrm{B}}\left(B_{0}-B\right) / B_{0} \Delta\right]\right\}^{-1}$. The broadening of the MO at $T \neq 0$ is entirely dictated by the behaviour of $\mathcal{F}_{0}$. This can be seen considering that, by the properties of arctangent, equation (8) is equivalent to [54]

$$
M_{T}=\left\{\begin{array}{ll}
M+A\left(1-\mathcal{F}_{0}\right) & 1 / B<1 / B_{0} \\
M-A \mathcal{F}_{0} & 1 / B>1 / B_{0}
\end{array},\right.
$$

where $M=A \arctan \left\{\cot \left[\pi \omega\left(\frac{1}{B}+\Delta\right)\right]\right\} / \pi$ is the magnetization at zero temperature. Notice the change of sign in the exponential, which is consistent with the limit $M_{T} \rightarrow M$ 
if $T \rightarrow 0(\beta \rightarrow \infty)$. In figure 5 it is schematically plotted $M$ and $M_{T}$, as a function of $1 / B$, plus the exponential functions which give the temperature correction. From this we can identify two properties of the broadening due to the temperature: the shift $\delta$ of the extrema and the width $w$ from which $M_{T} \simeq M$. Both $\delta$ and $w$ depend on the temperature, and in general also depend on $B_{0}$ and $\mu$. They are obtained from the equations

$$
\begin{aligned}
& \frac{\partial M_{T}}{\partial B}\left(\frac{1}{B}=\frac{1}{B_{0}}-\delta\right)=0 \\
& \mathcal{F}_{0}\left(\frac{1}{B}=\frac{1}{B_{0}}-w\right) \ll 1
\end{aligned}
$$

The first equation can only be solved numerically. In this way one obtains $\delta=\delta\left(T, B_{0}\right)$, and in general, for the same temperature, $\delta\left(T, B_{01}\right) \neq \delta\left(T, B_{02}\right)$. This dependence of $\delta$ with the magnetic field implies a broken periodicity of the MO with $1 / B$ at very low temperatures, although usually one has $\delta\left(T, B_{01}\right)-\delta\left(T, B_{02}\right) \ll 1 / \omega$. Nevertheless, as we will show later, when the temperature is increased one needs to consider the effect of the surrounding peaks, in which case the shift reaches the limit $\delta \rightarrow 1 / 4 \omega$, equal to the medium of the maxima and zero of the MO peaks. On the other hand,

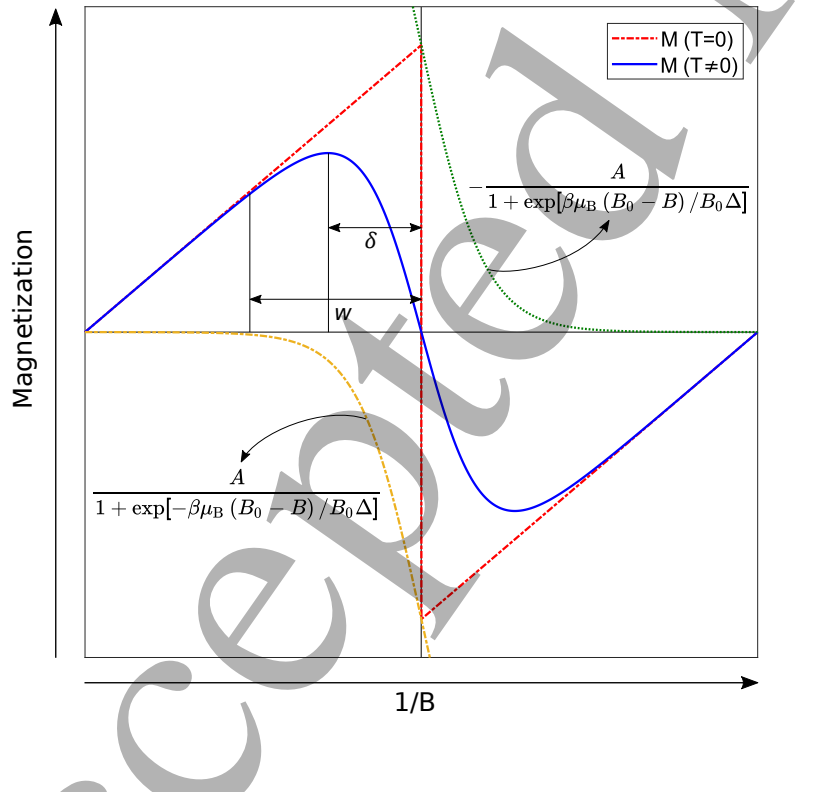

Figure 5. Schematic representation of the temperature effect over each MO peak, as expressed by equation (7). In red (dashed line) it is shown a magnetization peak at $T=0$, located at $1 / B_{0}$, and in blue (solid line) the magnetization at $T \neq 0$. It is also shown the exponentials that broaden the magnetization at non zero temperature, as described by equation (99). The peaks modification due to the temperature can be described by the parameters $w$ and $\delta$, as indicated. The width $w$ measures the reach of the temperature effect over each peak, while $\delta$ measures the extreme shift from its location at $T=0$. 
the width $w$ can be estimated from equation (11) by choosing a cutoff $\sigma \ll 1$ such that $\mathcal{F}_{0}\left(1 / B_{0}-w\right)=\sigma$. This gives a width $w \simeq \ln (1 / \sigma-1) k_{\mathrm{B}} T \Delta / \mu_{\mathrm{B}} B_{0}$, where from our experience it is sufficient to take $\sigma \sim 10^{-2}$ so $\ln (1 / \sigma-1) \sim 5$. The width $w$ is a measurement of the local influence of the temperature over each MO peak, and as such it will be fundamental in estimating the temperature limits corresponding to the different behaviours shown in figure 4.

\subsection{Spin splitting}

To study the influence of temperature over the observation of the spin splitting (SP) in the MO, we follow the same lines as we did in the graphene case 54, applying it to each of the frequencies now present. Then we consider two MO péaks with frequency $\omega$, at a given LL $n$, separated due to the SP, located in general at $1 / B_{1}=n / \omega-\Delta$ and $1 / B_{2}=n / \omega+\Delta$. From equation (7), the corresponding magnetization is

$$
M_{T}=\frac{A}{\pi} \sum_{s= \pm 1} \arctan \left\{\cot \left[\pi \omega\left(\frac{1}{B}+s \Delta\right)+\pi \mathcal{F}_{12}\right]\right\} \text {, }
$$

with $\mathcal{F}_{12}=\sum_{n=1,2}\left\{1+\exp \left[\beta \mu_{\mathrm{B}}\left(B_{n}-B\right) / \Delta B_{n}\right]\right\}^{-1}$. We know, from figure 5, that the width $w$ of this exponentials determines the observation of the MO at non zero temperature. Thus, for two peaks separated by $2 \Delta$ due to the SP, one would expect to see the SP in the MO only if $w<2 \Delta$. In fact, this result can be easily visualized by plotting equation (12) and the corresponding exponentials, as done in the figure 5 . This was done in graphene [54], where one observes that as $w$ approaches $2 \Delta$, the SP disappears in the MO, and one is left with one oscillation around the middle of the peaks. Consequently, given that $w \simeq 5 k_{\mathrm{B}} T \Delta / \mu_{\mathrm{B}} B$, from the condition $w=2 \Delta$ we get the spin temperature

$$
T_{s} \simeq \frac{2 \mu_{\mathrm{B}} B}{5 k_{\mathrm{B}}}
$$

where $B=\omega / n$ is the middle of two peaks separated due to the SP. The condition to observe the spin splitting in the MO is that $T<T_{s}$, which in order of magnitude means that the thermal energy $k_{\mathrm{B}} T$ is lower than the Zeeman energy $2 \mu_{\mathrm{B}} B$. This is the same temperature that was found in graphene, which is expected because $T_{s}$ depends only on the spin splitting effect in the MO and not on the broken valley degeneracy that appears in 2D buckled crystals. Moreover, it does not depend on the particular 2D material properties, such as $v_{\mathrm{F}}, l$ or $\lambda_{\mathrm{SO}}$, which again is expected because the $\mathrm{SP}$ alters the energy levels by the introduction of the crystal independent Zeeman term $2 \mu_{\mathrm{B}} B$. In the particular case considered in figure 4 , we get that for $1 / B \sim 1.11 / \mathrm{T}$, we have $T_{s} \sim 0.25 \mathrm{~K}$, so for the region of magnetic fields considered, one would not observe the $\mathrm{SP}$ in the $\mathrm{MO}$ at $T>0.25 \mathrm{~K}$. This is consistent with Figs. 4(b) and 4(c), where at $T=0.1 \mathrm{~K}<T_{s}$ we see the $\mathrm{SP}$, but at $T=0.1 \mathrm{~K}>T_{s}$ we do not. 


\subsection{Valley mixing}

We call the valley mixing (VM) effect in the $\mathrm{MO}$ to the mixing pattern that appears around the MO minimum, as can be seen in figures 4(a)-(c). This effect is independent of the SP and is caused by the broken valley degeneracy, which overlaps the MO peaks with different frequency. The way this overlap is produced determines how is the resulting mixing behaviour. To understand this, consider the minimum condition, when the peaks with frequency $\omega_{1}$ and $\omega_{2}$ are between one another (destructive interference), as separated as possible. This is shown in figure 6, where on the left we plotted the peaks with frequency $\omega_{1}$ (dashed line) and $\omega_{2}$ (solid line), and on the right the resulting MO obtained by their summation (for the sake of simplicity, we shall omit the SP of each peak, but the result obtained is independent of it). On the left, the vertical lines in

(a)

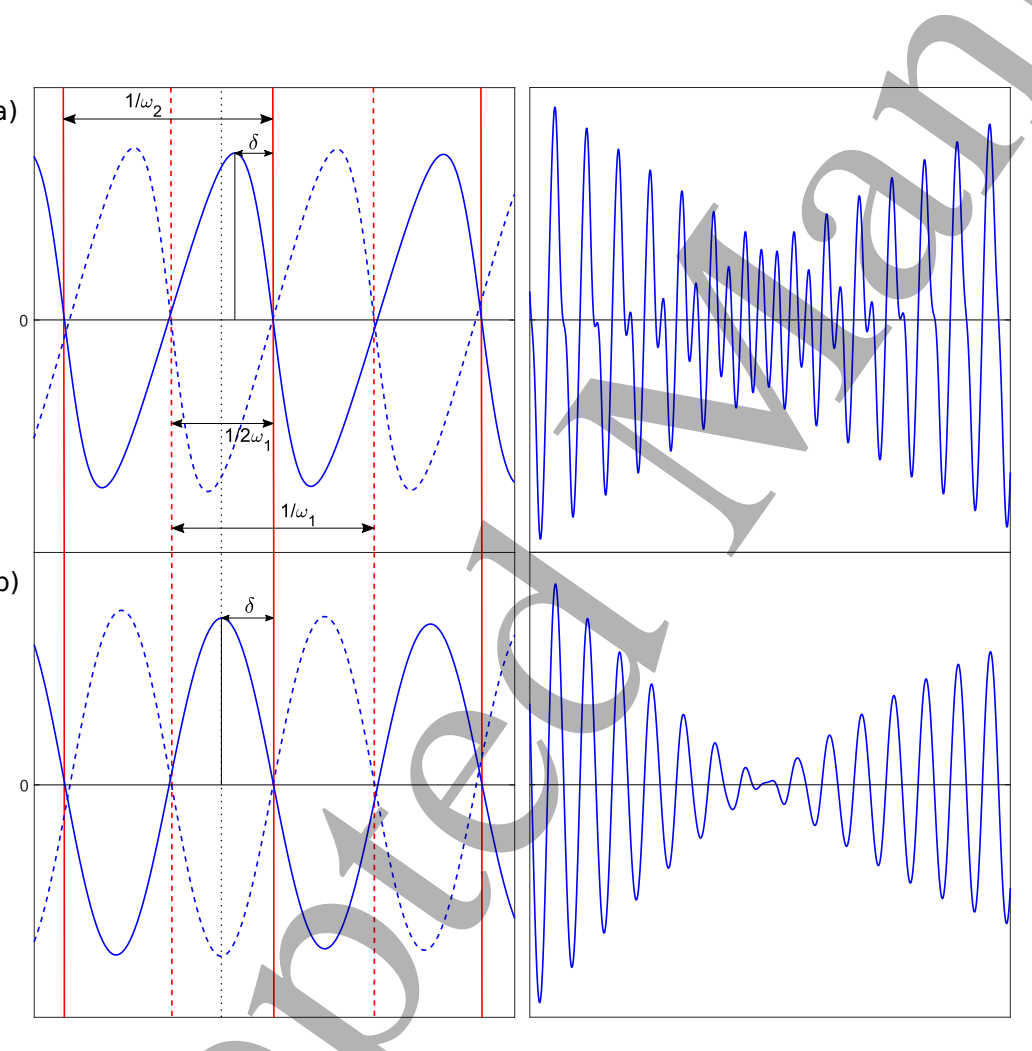

Figure 6. Relationship between the valley mixing (VM) in the MO around the beating minimum, and the extrema shift $\delta$ of the MO peaks. On the left it is shown two MO peaks $M_{1}$ (dashed line) and $M_{2}$ (solid line), with frequencies $\omega_{1}$ and $\omega_{2}$, as a function of $1 / B$, where the vertical lines in red correspond to the peaks location at $T=0$ while the blue lines are the $\mathrm{MO}$ at $T \neq 0$. The region of the plot corresponds to the minimum location in the MO, where there is destructive interference between the peaks. In the right it is shown the resulting $\mathrm{MO}$ around that minimum location, obtained by the sum of the $M_{1}$ and $M_{2}$. The case in (a) corresponds to a shift $\delta<1 / 4 \omega$, for which the minimum and maximum of $M_{1}$ and $M_{2}$ are not in the same location, causing the mixing pattern seen in the resulting MO on the right. On the other hand, when the shift reaches the limit $\delta=1 / 4 \omega$ in (b), the minimum and maximum of $M_{1}$ and $M_{2}$ are approximately in the same location, in which case there is no VM in the MO around the beating minimum. 
red correspond to the peaks locations at $T=0$, with their periodicity $1 / \omega$ indicated for each case. The first situation, figure 6(a), corresponds to the case where the MO show a VM behaviour around the minimum, as can be seen in the right figure. This can be explained by analysing how the resulting MO is obtained from the summation of the peaks shown in the left figure. There we see that, at this temperature, the MO extrema shift $\delta$ is less than $1 / 4 \omega$ (black dot line), so the maximum and minimum are not in the same location, and therefore the MO do not become zero. On the other hand, when the temperature increases, the extrema shift $\delta$ reaches the limit $1 / 4 \omega$ for both peaks, as shown in figure 6(b), in which case the maximum and minimum are approximately in the same location. This behaviour is maintained if the temperature is further increased, for the shift $\delta$ remains at $1 / 4 \omega$ and the increasing of the temperature only reduces the amplitude of the oscillations. Therefore, the condition to observer the VM in the MO is that the extrema shift $\delta$ is less than $1 / 4 \omega$.

For the MO $M_{i, s}$, with frequency $\omega_{i}$ and phase $s \Delta_{i}$, the extrema shift $\delta$ of a peak at $1 / B_{l}=l / \omega_{i}-s \Delta_{i}$ is obtained from the equation $\partial M_{i, s} / \partial B\left[1 / B_{l}-p \delta_{p, l}\right]=0$, where $p=1(-1)$ for the maximum (minimum) shift. Then, using (7), the equation for obtaining $\delta$ becomes

$$
1=\frac{\beta \alpha^{2}}{8 \mu\left(1 / B_{l}-p \delta_{p, l}\right)^{2}} \sum_{n} \frac{1}{B_{n}} \operatorname{sech}^{2}\left[\frac{\beta \alpha^{2} \omega_{i}}{4 \mu}\left(\frac{1}{B_{l}}-\frac{1}{B_{n}}-p \delta_{p, l}\right)\right] .
$$

The equation (14) can be solved numerically for each $B_{l}$ as a function of the temperature, obtaining that $\delta$ follows an exponential distribution, with the limit $\delta \rightarrow 1 / 4 \omega_{i}$. The temperature at which we get this limit can be estimated from the relation of $\delta$ with the width $w$ of the exponential associated with the peak at $B_{l}$, obtained from equation (11). This was done in figure 7, where we show the numerical solution of equation (14) for $\delta$, and the width $w=5 k_{\mathrm{B}} T \Delta_{i} / \mu_{\mathrm{B}} B_{0}$ (considering $\mathcal{F}_{0}\left(1 / B_{0}-w\right)=\sigma$ with $\left.\sigma \sim 10^{-2}\right)$. The values correspond to silicene, with $\mu=0.25 \mathrm{eV}$, el $E_{z}=92 \mathrm{meV}$, and considering the spin up peak with frequency $\omega_{1}=137.53 \mathrm{~T}$ at $l=172$, which gives $1 / B_{l} \simeq 1.25$ $1 / \mathrm{T}$ (thus the shift and width calculated correspond to the peak around the minimum in figure (4). Then we can see, in figure 7, that not only $\delta$ tends to the limit $1 / 4 \omega_{1}$, but also that when it does it $w \gtrsim 1 / \omega_{1}$. Hence, referring to figure 5 , for a given peak the extrema shift $\delta$ approximately reaches its limit value when the width $w$ is about the period of oscillation $1 / \omega$. This gives an estimation for the temperature $T_{v}$ at which $\delta \rightarrow 1 / 4 \omega$, for then $w \sim 1 / \omega$ and therefore

$$
T_{v} \simeq \frac{\hbar v_{\mathrm{F}}^{2} e B}{5 \mu k_{\mathrm{B}}}
$$

Then, following figure 6, $T_{v}$ is also the temperature at which the valley mixing would not longer be seen in the MO. In that case it should be noted that the magnetic field that goes into equation (15) corresponds to the peaks at the destructive interference, or the absolute minima, that is $1 / B=(r+1 / 2) /\left(\omega_{1}-\omega_{2}\right)$ with $r$ an integer. For the particular case of figure 4 we get $T_{v} \simeq 1.48 \mathrm{~K}$. This is in agreement with figure $4(\mathrm{~d})$, 


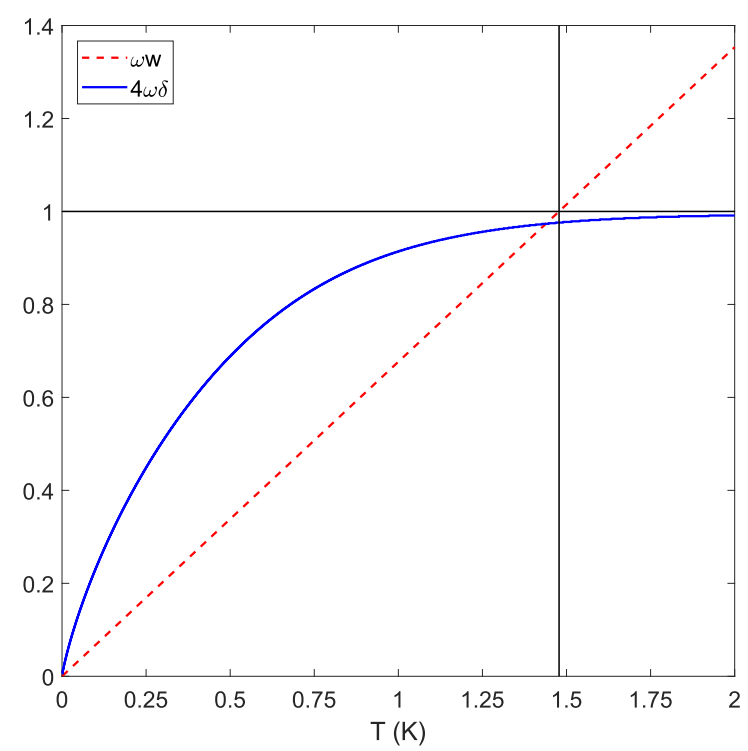

Figure 7. Relationship between the extreme shift $\delta$ and the width $w$ of a MO peak, as defined in figure 5, for silicene, with $\mu=0.25 \mathrm{eV}$ and $e l E_{z}=92 \mathrm{meV}$, considering the peak with frequency $\omega=\omega_{1} \simeq 137.5 \mathrm{~T}$ at $1 / B_{0}=172 / \omega_{1}-\Delta_{1}=1.251 / \mathrm{T}$. In the red dashed line it is plot $\omega w$, where $w=5 T \Delta / \mu_{\mathrm{B}} B_{0}$ is the width of the exponential associated with the peak at $1 / B_{0}$, as considered in equation (7). In the blue solid line it is plot $4 \omega \delta$, where $\delta$ is the maximum shift obtained numerically from equation (14), with $B_{l}=B_{0}$ and $p=1$. As we see, $\delta$ tends exponentially to $1 / 4 \omega$, and when it does it we have $w \gtrsim 1 / \omega$. In other words, the maximum shift reaches its limit value when the range of the temperature influence over each MO peaks is bigger than the peaks separation $1 / \omega$.

where for $T=1.5 \mathrm{~K}$ one does not see the $\mathrm{VM}$ in the MO. It is interesting to compare the valley temperature $T_{v}$ with the spin temperature $T_{s}$ given by equation (13). We have $T_{s} / T_{v}=2 \mu_{\mathrm{B}} \mu / \hbar v_{\mathrm{F}}^{2} e$, which from equations (4) and (5) implies $T_{s} / T_{v}=2 \Delta \omega$. Thus the ratio between these two temperatures is equal to the ratio between the period $1 / \omega$ and phase difference $2 \Delta$ of the peaks (and this ratio is equal for all peaks). Of course, this is an expected result because each temperature was calculated from the width given by equation (11), with $w=2 \Delta$ for $T_{s}$ and $w=1 / \omega$ for $T_{v}$.

\subsection{High temperature MO approximation}

When $T>T_{v}$, we can say the fine structure of the MO is damped by the temperature, and one is left with oscillations whose extrema, for each frequency, are always shifted $1 / 4 \omega$ from the peaks locations at $T=0$. In this situation it becomes more convenient to describe the MO using the LK formula given by equation (6), for then we can approximate $\sinh (\lambda p) \sim \exp (-\lambda p)$. Indeed, this approximation implies $\exp (-\lambda p) \ll 1$ or $\lambda p \gg 1\left(\lambda=4 \pi^{2} \mu k_{\mathrm{B}} T / \alpha^{2} B\right)$, which is satisfied for all $p$ if $T \gg \hbar v_{\mathrm{F}}^{2} e B / 2 \pi^{2} \mu k_{\mathrm{B}}=$ $5 T_{v} / 2 \pi^{2} \simeq T_{v} / 4$. Thus it is good approximation to take $\sinh (\lambda p) \sim \exp (-\lambda p)$ if 
$T>T_{v}$. Then the summation over $p$ in equation $(6)$ can be easily evaluated to $\lambda \sin \left[2 \pi \omega_{i}\left(1 / B+s \Delta_{i}\right)\right] \cosh (\lambda)$, where we used the fact that in this regime $\cosh (\lambda) \gg 1$ so $\cosh (\lambda)+\cos \left[2 \pi \omega_{i}\left(1 / B+s \Delta_{i}\right)\right] \simeq \cosh (\lambda)$. We can further approximate the expression for $M_{T}$ by noticing that in this high temperature regime, the difference between the amplitudes $A_{i}$ is practically negligible, so we can use the amplitude $A_{p} / 4$, where $A_{p}=2 \sum_{i=1,2} A_{i} \simeq 2\left[\lambda_{\text {SO }}^{2}+\left(e l E_{z}\right)^{2}-\mu^{2}\right] / \phi \mu$. Then, rewriting the sine summation in equation (6), we get the result

$$
\begin{aligned}
M_{T} & \simeq A_{p} \frac{k_{\mathrm{B}} T \gamma}{B} \operatorname{sech}\left(\frac{\pi k_{\mathrm{B}} T \gamma}{B}\right) \cos \left(\gamma \mu_{\mathrm{B}}\right) \\
& \times \sin \left[\frac{\pi\left(\omega_{1}+\omega_{2}\right)}{B}\right] \cos \left[\frac{\pi\left(\omega_{1}-\omega_{2}\right)}{B}\right],
\end{aligned}
$$

where we defined $\gamma \equiv 2 \pi \mu / \hbar v_{\mathrm{F}}^{2} e$. It is instructive to analyse each term in equation (16). The temperature effect is entirely contained in the term $\left(k_{\mathrm{B}} T \gamma / B\right) \operatorname{sech}\left(\pi k_{\mathrm{B}} T \gamma / B\right)$, which as expected goes to zero as $T$ increases, and it acts by just reducing the overall amplitude of the MO. In other words, in this regime the temperature does not modify the shape of each MO peak, which of course is expected, as we are at temperatures such that we already reached the limit $\delta \rightarrow 1 / 4 \omega$ for all $B$ considered. The term $\cos \left(\gamma \mu_{\mathrm{B}}\right)$ is independent of the magnetic field and contains the effect due to the SP, which then only acts as a reduction factor in the MO amplitude. Lastly, the last two trigonometric functions in equation (16) give the MO profile. Under the beating condition $\left(\omega_{1}-\omega_{2}\right) / \omega_{1} \ll 1$, the first term causes the internal, small period oscillations, whereas the second acts as the envelope of the internal oscillations. This separation between each contribution will be particular useful in order to obtain the MO envelope.

\subsection{MO envelope}

We shall now obtain an expression for the MO envelope, restricting ourselves to the beating condition, so that $\left(\omega_{1}-\omega_{2}\right) / \omega_{1} \ll 1$. In the general case, at a given temperature one should numerically obtain the shift $\delta$ as function of $B$, and from it construct the MO envelope, as was done in graphene [54]. The generalization to 2D materials with broken valley degeneracy is trivially done by taking into account the two frequencies involved and the resulting beating phenomenon. For simplicity we will omit this transition region and consider only the case of high temperatures, such that $\delta=1 / 4 \omega$. This implies $T>T_{v}$ for all the magnetic field considered, in which case it is convenient to work with equation (16) for the MO. To obtain its envelope we just have to eliminate the internal oscillations in the sine function by evaluating it at its maximum value. Thus we get envelope

$$
E \simeq A_{p} \frac{k_{\mathrm{B}} T \gamma}{B} \operatorname{sech}\left(\frac{\pi k_{\mathrm{B}} T \gamma}{B}\right) \cos \left(\gamma \mu_{\mathrm{B}}\right) \cos \left[\frac{\pi\left(\omega_{1}-\omega_{2}\right)}{B}\right] .
$$

The fact that the envelope is obtained when $\sin \left[\pi\left(\omega_{1}+\omega_{2}\right) / B\right]=1$ implies that the extreme shift for the internal oscillations is of the form $1 / B=2 l /\left(\omega_{1}+\omega_{2}\right)-$ 
$1 / 2\left(\omega_{1}+\omega_{2}\right)$, which resembles $1 / B=l / \omega-\delta$ with $\omega=\left(\omega_{1}+\omega_{2}\right) / 2$ and $\delta=1 / 4 \omega$. Thus we get our previous result that at this regime the extreme shift is equal to $1 / 4 \omega$, with the frequency being the average between $\omega_{1}$ and $\omega_{2}$. From equation (17) we can also get the temperature decay envelope $E_{d}=E / \cos \left[\pi\left(\omega_{1}-\omega_{2}\right) / B\right]$. In figure 8 it is shown the MO and its envelope in germanene, for $T=3 \mathrm{~K}$ with $e l E_{z}=25 \mathrm{meV}$ and $\mu=0.25 \mathrm{eV}$. In this case, from equation 15 we have $T_{v} \simeq 2.6 \mathrm{~K}$ for $1 / B=0.5$ $1 / \mathrm{T}$. Hence $T>T_{v}$ for all the $B$ considered and therefore the MO and its envelope are given by equations (16) and (17). It should be noted that due to the temperature, not only the oscillation amplitude is damped, but also there is a shift of each MO beating maximum from its location at $T=0$. At zero temperature, this maximum occurs at $1 / B_{M}=r /\left(\omega_{1}-\omega_{2}\right)$, with $r$ an integer (see figure 3), but at non zero temperature, the new maximum occurs when $\partial E / \partial B=0$, and because the temperature decay $E_{d}$ depends on $B$, then its solution is no longer $1 / B_{M}$. This can be seen in the zoomed oscillations in figure 8, where the decay envelope $E_{d}$ (calculated considered the maxima at $\left.1 / B_{M}=r /\left(\omega_{1}-\omega_{2}\right)\right)$ does not exactly pass over the MO extrema. On the other hand, the zeros in the MO are fixed at $(r+1 / 2) /\left(\omega_{1}-\omega_{2}\right)$, the same location of the beating minimum at $T=0$. From an experimental point of view, this is an useful result because the distance between the MO nodes will be always the beating period

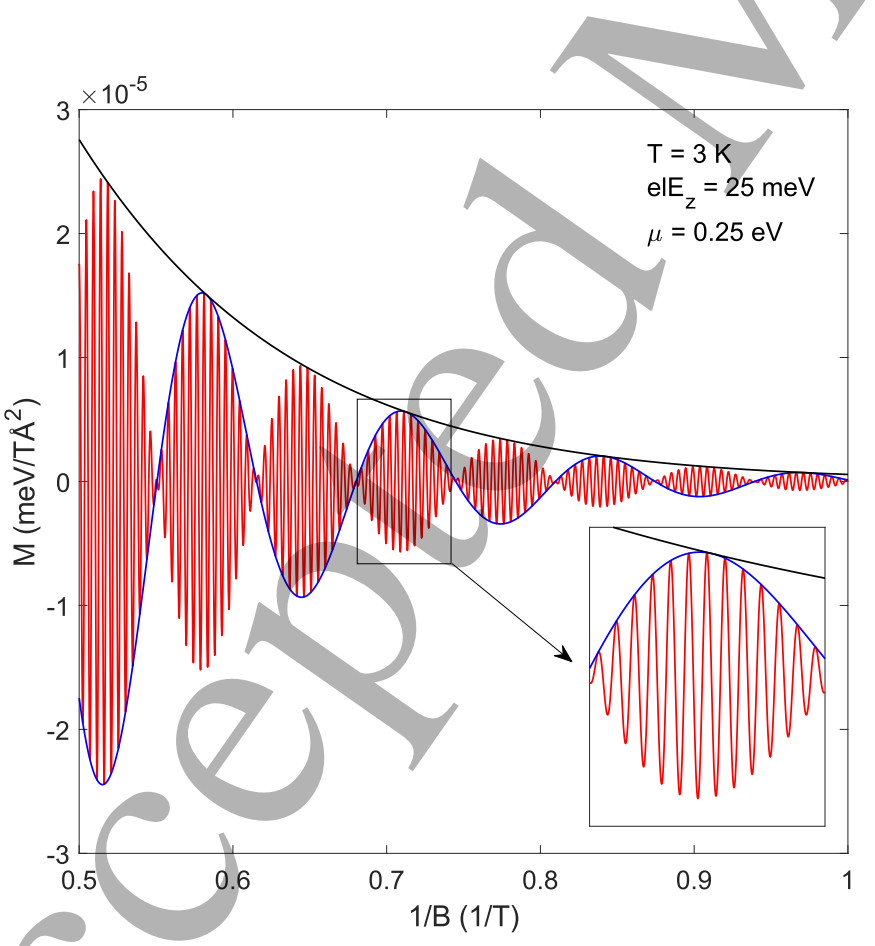

Figure 8. Magnetic oscillations in germanene, for $T=3 \mathrm{~K}$ with el $E_{z}=25 \mathrm{meV}$ and $\mu=0.25 \mathrm{eV}$, such that $\left(\omega_{1}-\omega_{2}\right) / \omega_{1} \simeq 0.07$. In this situation, the fine structure of the MO is lost, given that from equation 15 we have $T_{v} \simeq 2.6 \mathrm{~K}$ for $1 / B=0.5$ $1 / \mathrm{T}$. Thus the $\mathrm{MO}$ can be expressed with the Eq (16). The beating envelope $E$ in blue is given by equation (17), while the temperature decay envelope $E_{d}$ in black is $E=E_{d} \cos \left[\pi\left(\omega_{1}-\omega_{2}\right) / B\right]$. 
$1 /\left(\omega_{1}-\omega_{2}\right)=\hbar v_{\mathrm{F}}^{2} / 2 \lambda_{\mathrm{SO}} l E_{z}$. Thus, independently of the temperature, by measuring when the MO is zero one can obtain information about the crystal parameters.

\section{Conclusions}

We studied the magnetic oscillations (MO) in 2D materials with a honeycomb buckled structure, in the low energy approximation, where the electrons are described by a Dirac-like Hamiltonian. Examples of these system are silicene, germanene, stanene and phosphorene. We considered a perpendicular electric and magnetic field, taking into account the spin orbit interaction (SOI) and the Zeeman effect. For a constant positive Fermi energy, we showed that, at zero temperature, the MO can be decomposed as the sum of four sawtooth oscillations ( $\mathrm{SO}$ ), associated with the change of valley and spin in the last energy level occupied. The four $\mathrm{SO}$ consist of two unique frequencies, each one with two different phases due to the spin splitting. The frequencies depend on the crystals properties, as well as the Fermi energy and electric field, and the corresponding oscillation occurs only if they are positive. Hence, depending on the values of the Fermi energy and electric field, one can have only one frequency in the MO, or directly no oscillation. When both frequencies are present, the MO show an interference pattern. A beating phenomenon is seen only if the frequencies are close, which results in a rombolike pattern in the MO at zero temperature. When the frequencies are further apart, the MO show a more disperse, non-beating pattern, where the behaviour depends specifically on the values of the frequencies. We studied the condition to observe a beating in the MO, obtaining that the lower the Fermi energy, the lower the perpendicular electric field needs to be.

At non zero temperature, we considered the broadening of the MO using two different approaches. One was the Lifshitz-Kosevich (LK) formula that considers the temperature effect by the introduction of a reduction factor. The other approach, recently developed in graphene, considers the temperature effect by local corrections over each MO peak and thus is particular useful at low temperatures. Using this last approach we studied how the increase of the temperature alters the observation of the fine structure of the $\mathrm{MO}$, due to the valley and spin. We showed that this can be related to the width of the Fermi-Dirac like functions that modify each magnetization peak at non zero temperature. Specifically, we obtained that in order to observe the spin splitting (SP), the width must be lower than the MO phase difference. Likewise, in order to observe valley mixing (VM) effects in the MO, the width must be lower than the MO period. When the temperature is such that the SP and VM are no longer seen, then the MO is best described by the LK formula, for one can approximate and easily evaluate the series. We then obtained a simple expression for the MO, and its envelope, where one can clearly see how the different frequencies produce a beating phenomenon.

The results obtained show unique properties in the MO in 2D materials. The interplay between the valley and spin, under a perpendicular electric field, gives rise to oscillations with different frequencies and phases, a behaviour not seen in conventional 


\section{Temperature effect on the magnetic}

oscillations in $2 D$ materials

metals. Because of this, by studying the shape of the MO one can obtain information about the 2D materials parameters. For instance, we showed the presence of zeros in the $\mathrm{MO}$ when there is a beating phenomenon, and the temperature is high enough such that the SP and VM are not longer appreciable. This may be useful from an experimental point of view, for the location of these zeros depends exclusively on the perpendicular electric field and the crystals properties, such as the Fermi velocity, buckle height and SOI. Lastly, we want to remark that the results obtained correspond to the pristine case, where no effect of impurities is considered. It is known that the impurities also broaden the MO, so one would expect a similar behaviour to the one described for the temperature. In particular, the higher the impurities concentration, the lower the temperature necessary to observe the fine structure of the MO. On the other hand, an in-plane electric field would also be expected to modulate the MO, as has been reported in graphene.

\section{Acknowledgments}

This paper was partially supported by grants of CONICET (Argentina National Research Council) and Universidad Nacional del Sur (UNS) and by ANPCyT through PICT 2014-1351. Res. N 270/15. N: 2014-1351, and PIP 2014-2016. Res. N 5013/14. Código: 11220130100436CO research grant, as well as by SGCyT-UNS., J. S. A. and P. J. are members of CONICET., F. E. acknowledge research fellowship from this institution.

\section{Appendix A. MO at zero temperature}

We will derive equation (2) for the magnetic oscillations at $T=0$, for the general case of a $2 \mathrm{D}$ crystal with energy levels $\varepsilon_{\zeta, n, \eta, s}=\zeta\left[\left(s \lambda_{\mathrm{SO}}-\eta e l E_{z}\right)^{2}+\alpha^{2} n B\right]^{1 / 2}-s \mu_{\mathrm{B}} B$, where $\zeta= \pm 1$ for the conduction and valence bands, $\alpha=v_{\mathrm{F}} \sqrt{2 \hbar e}, n=0,1,2, \ldots$ for the Landau level (LL) and $\eta, s= \pm 1$ are the valley and spin indices. Graphene is a special case, with $\lambda_{\mathrm{SO}} \simeq 0$ and $l=0$, and therefore the derivation of $M$ will follow an analogous procedure to the one employed in [54]. We shall repeat the essential steps of this derivation just for completeness.

We consider a constant Fermi energy $\mu>0$, such that at zero temperature the valence band is full while the conduction band is partially filled. We will note the conduction energy levels $\varepsilon_{m}=\left[\left(s_{m} \lambda_{\mathrm{SO}}-\eta_{m} e l E_{z}\right)^{2}+\alpha^{2} n_{m} B\right]^{1 / 2}-s_{m} \mu_{\mathrm{B}} B$, where we have introduced the decreasing energy sorting index $m=0,1,2, \ldots$, so $n_{m}$ gives the LL, $\eta_{m}$ the valley and $s_{m}$ the spin for the $m$ position. At a given $\mu>0$, all energy levels $m=0,1,2, \ldots, f$ are filled, where $f$ is such that $\varepsilon_{f} \leq \mu<\varepsilon_{f+1}$. Then the grand potential at zero temperature is $\Omega=\Omega_{V}+\sum_{m=0}^{f} D\left(\varepsilon_{m}-\mu\right)$, where $\Omega_{V}$ is the grand potential due to the filled valence band. It is important to notice that the oscillation in $\Omega$ is caused only by the last term, due to the conduction band, associated with the change in the last energy level as $B$ is changed. On the other hand, the first term $\Omega_{V}$ 
Temperature effect on the magnetic oscillations in $2 D$ materials

makes a non-oscillatory contribution since the valence band is always filled for $\mu>0$. Separating $\varepsilon_{m}=\varepsilon_{m}^{0}-\varepsilon_{m} \mu_{\mathrm{B}} B$, with $\varepsilon_{m}^{0}=\left[\left(s_{m} \lambda_{\mathrm{SO}}-\eta_{m} e l E_{z}\right)^{2}+\alpha^{2} n_{m} B\right]^{1 / 2}$, we can write the conduction grand potential as

$$
\Omega_{C}=\Omega_{0}-B M_{P}
$$

where $\Omega_{0}=\sum_{m=0}^{f} D\left(\varepsilon_{m}^{0}-\mu\right)$ and $M_{P}=\mu_{\mathrm{B}} D \sum_{m=0}^{f} s_{m}$ is the Pauli paramagnetism associated with the spin population. The conduction magnetization is given by $M_{C}=-\mathcal{A}^{-1}\left(\partial \Omega_{C} / \partial B\right)_{\mu}$, where $\mathcal{A}$ is the sheet area. Deriving and regrouping we get

$$
M_{C}=-\frac{1}{2 B}\left(3 \frac{\Omega_{C}}{\mathcal{A}}+\rho \mu\right)+M^{\prime}+\frac{1}{2} m_{P},
$$

where $\rho=N / \mathcal{A}=\sum_{m=0}^{f} D / \mathcal{A}=A(f-1) / D$ is the density of conduction electrons, $m_{p}=M_{P} / \mathcal{A}$ and

$$
M^{\prime}=\frac{e}{2 h} \sum_{m=0}^{f} \frac{\left(s_{m} \lambda_{\mathrm{SO}}-\eta_{m} e l E_{z}\right)^{2}}{\varepsilon_{m}+s_{m} \mu_{\mathrm{B}} B} .
$$

It is worth noting that, looking at equation (A.3), we see that $M^{\prime}$ is related to the SOI and the buckle height. Thus this contribution is zero in graphene, while in the other Dirac crystals it becomes appreciable, especially at large electric field. From equation A.2 we directly see that the MO have a sawtooth oscillation (SO) produced whenever $\rho, M^{\prime}$ or $m_{P}$ change discontinuously, $\Omega_{C}$ being continuous always. The SO amplitude $\Delta M$ is given by

$$
\Delta M=-\frac{\mu}{2 B} \Delta \rho+\Delta M^{\prime}+\frac{1}{2} \Delta m_{P}
$$

where each contribution $\Delta \rho, \Delta M^{\prime}$ and $\Delta m_{P}$ is determined by the discontinuous change in the parameters $n_{f}, \eta_{f}$ and $s_{f}$ which define the last energy level occupied. The SO peaks occur at $B_{i}$ such that $\varepsilon_{f}\left(B_{i}\right)=\mu$. Therefore $\mu=$ $\left[\left(s_{i} \lambda_{\mathrm{SO}}-\eta_{i} e l E_{z}\right)^{2}+\alpha^{2} n_{i} B_{i}\right]^{1 / 2}-s_{i} \mu_{\mathrm{B}} B_{i}$, and given that usually $\mu_{\mathrm{B}} B / \mu \ll 1$, we obtain

$$
\frac{1}{B_{i}}=\frac{n_{i} \alpha^{2}-2 s_{i} \mu \mu_{\mathrm{B}}}{\mu^{2}-\left(s_{i} \lambda_{\mathrm{SO}}-\eta_{i} e l E_{z}\right)^{2}} .
$$

From this we can consider four types of MO peaks, taking into account the possible changes of LL, valley and spin. Each peak is associated to a fixed valley and spin, with its oscillation being caused when the LL changes by one. The period of oscillation is $\Delta(1 / B)=1 / B_{2}-1 / B_{1}$, with $\Delta n=n_{2}-n_{1}=1$, while $\eta=\eta_{1}=\eta_{2}$ and $s=s_{1}=s_{1}$. Consequently, from equation A.5 we obtain the period $\Delta(1 / B)$ and frequency $\omega=[\Delta(1 / B)]^{-1}$

$$
\omega_{\eta s}=\frac{\mu^{2}-\left(s \lambda_{\mathrm{SO}}-\eta e l E_{z}\right)^{2}}{\alpha^{2}} .
$$




\section{Temperature effect on the magnetic oscillations in $2 D$ materials}

Then we can write equation A.5 as $1 / B_{\eta s}\left(n_{i}\right)=n_{i} / \omega_{\eta s}+\Delta_{\eta s}$, where $\Delta_{\eta s}$ is the phase

$$
\Delta_{\eta s}=-\frac{2 s_{i} \mu \mu_{\mathrm{B}}}{\mu^{2}-\left(s \lambda_{\mathrm{SO}}-\eta e l E_{z}\right)^{2}} .
$$

From equations A.6 and A.7 we see that $\omega_{K \uparrow}=\omega_{K^{\prime} \downarrow}, \omega_{K \downarrow}=\omega_{K^{\prime} \uparrow}$ while $\Delta_{K \uparrow}=$ $-\Delta_{K^{\prime} \downarrow}, \Delta_{K^{\prime} \uparrow}=-\Delta_{K \downarrow}$, so there are two unique frequencies and phases. Under the conditions assumed, the peaks can only occur if $\omega_{\eta s}>0$ in equation (A.6). Indeed, remember that equation A.6 was derived from equation A.5 considering $B_{1}$ and $B_{2}$ such that $\varepsilon_{1}=\mu=\varepsilon_{2}$, with $n_{2}>n_{1}$. Thus, if $\omega_{\eta s}<0$ then $\mu^{2}<\left(s \lambda_{\text {SO }}-\eta e l E_{z}\right)^{2}$, which for $\mu_{\mathrm{B}} B / \mu \ll 1$ implies $2 \varepsilon_{i} s \mu_{\mathrm{B}}>\alpha^{2} n_{i}$. Therefore we have $2\left(\varepsilon_{2}-\varepsilon_{1}\right) s \mu_{B}>$ $\alpha^{2}\left(n_{2}-n_{1}\right)$, but $\varepsilon_{2}-\varepsilon_{1}=0$, so $0>\alpha^{2}\left(n_{2}-n_{1}\right)$. This result means $n_{2}<n_{1}$, in contradiction with the initial assumption of $n_{2}>n_{1}$.

The peaks amplitude $A_{\eta s}$ is obtained from equation (A.4). Suppose the magnetic field is increased so the last sorted position $f$ changes to $f-1$. For $\Delta \rho$ and $\Delta m_{P}$ we easily get $\Delta \rho=D / \mathcal{A}=B / \phi$ and $\Delta m_{P}=D \mu_{\mathrm{B}} s_{f} / \mathcal{A}=B \mu_{\mathrm{B}} s_{f} / \phi$. For $\Delta M^{\prime}$, when the change is produced we have $\varepsilon_{f}=\mu$, so from equation (A.3) we get $\Delta M^{\prime}=\left(s_{f} \lambda_{\mathrm{SO}}-\eta_{f} e l E_{z}\right)^{2} / 2 \phi\left(\mu+s_{f} \mu_{\mathrm{B}} B\right)$. Thus in general

$$
\begin{aligned}
A_{\eta s} & =\frac{e}{2 h}\left[\frac{\left(s \lambda_{\mathrm{SO}}-\eta e l E_{z}\right)^{2}}{\mu+s \mu_{\mathrm{B}} B}-\mu+s \mu_{\mathrm{B}} B\right] \\
& \simeq-\frac{e}{2 h} \frac{\omega_{\eta s}}{\mu},
\end{aligned}
$$

where we consider $\mu_{\mathrm{B}} B / \mu \ll 1$. We are now in position to express the four SO, whose amplitude, frequency and phase are obtained from equations (A.6), (A.7), (A.8). Each type of peak can be expressed as an infinite series, so the SO are written as

$$
M_{\text {saw }}=\sum_{\eta s} A_{\eta s} \sum_{p=1}^{\infty} \frac{1}{\pi p} \sin \left[2 \pi p \omega_{\eta s}\left(\frac{1}{B}-\Delta_{\eta s}\right)\right],
$$

The equation A.9 gives the SO contribution to the MO. There is still another oscillatory contribution, which comes from the continuous oscillation in $\Omega_{C}$. From equation A.9 we see that $\Omega_{C}^{o s c}$ should be of the form $\Omega_{C}^{o s c}=$ $\sum_{\eta s} C_{\eta s} \sum_{p=1}^{\infty} \cos \left[2 \pi p \omega_{\eta s}\left(\frac{1}{B}-\Delta_{\eta s}\right)\right] /(\pi p)^{2}$, where $C_{\eta s}$ is such that $M_{o s c}=$ $-\mathcal{A}^{-1}\left(\partial \Omega_{C}^{o s c} / \partial B\right)_{\mu}$. From equation $\mathrm{A} .9$ we get $C_{\eta s}=-\mathcal{A} B^{2} A_{\eta s} / 2 \omega_{\eta s}$, so the MO are given by

$$
\begin{aligned}
M_{o s c} & =\sum_{\eta s} A_{\eta s} \sum_{p=1}^{\infty} \frac{1}{\pi p} \sin \left[2 \pi p \omega_{\eta s}\left(\frac{1}{B}-\Delta_{\eta s}\right)\right] \\
& -\sum_{\eta s} A_{\eta s} \frac{B}{\omega_{\eta s}} \sum_{p=1}^{\infty} \frac{1}{(\pi p)^{2}} \cos \left[2 \pi p \omega_{\eta s}\left(\frac{1}{B}-\Delta_{\eta s}\right)\right],
\end{aligned}
$$

where we used the fact that $\partial A_{\eta s} / \partial B \simeq 0$ so $\partial C_{\eta s} / \partial B \simeq-\mathcal{A} B A_{\eta s} / \omega_{\eta s}$. The equation A.10 is in agreement with [38], where the oscillating part of the magnetization is 


\section{Temperature effect on the magnetic oscillations in $2 D$ materials}

written as an infinite series. There is still the non-oscillatory contribution to the magnetization, from both the valence and conducting band. Nevertheless, it can be shown [37] that when $\mu>\left|s \lambda_{\mathrm{SO}}-\eta e l E_{z}\right|$, this contribution cancels and the total magnetization is given by equation A.10. From equation A.6 we see that the condition $\mu>\left|s \lambda_{\mathrm{SO}}-\eta e l E_{z}\right|$ implies $\omega_{\eta s}>0$, which as discussed above is also the condition to observe the oscillation corresponding to the peak $\eta s$. Thus, because we will be mainly interested with the MO, we shall omit the non-oscillatory contribution and take the total magnetization given by equation A.10. It is worth noting that in this formalism the spin splitting due to the Zeeman effect is already taken into account in equation $\mathrm{A} .9$, so there is no need to introduce it as a reduction factor.

We can further simplify equation A.10 by noticing that the cosine series is usually much smaller than the sine series. This can be seen by analysing the corresponding series amplitude ratio, given by $A_{\eta s}^{\text {cos }} / A_{\eta s}^{s i n}=B / \omega_{\eta s}$. Considering that for all the $2 \mathrm{D}$ crystals we have $\alpha \sim 10 \mathrm{meV} / \sqrt{\mathrm{T}}$, and we will work with/values arøund $\mu \sim 10^{2} \mathrm{meV}$ and $\left|s \lambda_{\mathrm{SO}}-\eta e l E_{z}\right| \sim 10 \mathrm{meV}$, we have $A_{\eta s}^{\text {cos }} / A_{\eta s}^{\text {sin }} \sim 10^{-2} B[\mathrm{~T}]$. Therefore, unless $B$ is very high we can neglect the cosine series in equation A.10. Then the sine series can be easily evaluated to obtain the MO at zero temperature

$$
M=\sum_{\eta s} \frac{A_{\eta s}}{\pi} \arctan \left\{\cot \left[\pi \omega_{\eta s}\left(\frac{1}{B}-\Delta_{\eta s}\right)\right]\right\} .
$$

Finally, equation A.11 can be conveniently rewritten by separating the peaks with frequency $\omega_{1}=\omega_{K \uparrow}=\omega_{K^{\prime} \downarrow}$ and $\omega_{2}=\omega_{K \downarrow}=\omega_{K^{\prime} \uparrow}$, with phases $\Delta_{1}=\Delta_{K \uparrow}=-\Delta_{K^{\prime} \downarrow}$ and $\Delta_{2}=\Delta_{K^{\prime} \uparrow}=-\Delta_{K \downarrow}$, which leads to equation (2) for the MO at zero temperature.

\section{Appendix B. MO at non zero temperature}

We will derive the expression (7) for the MO at non zero temperature. As it was done in the zero temperature case, the derivation will follow the same approach already applied in graphene [54], which we shall repeat here for completeness. We start with the grand potential $\Omega_{T}$ at $T \neq 0$, for which we can use its non-relativistic expression in the absence of impurities [37, 157]. It is convenient to separate $\Omega_{T}$ by the contribution of each peak associated to the resulting MO. In other words, we separate $\Omega_{T}=\sum_{i=1,2, s= \pm 1} \Omega_{i, s}$, where

$$
\Omega_{i, s}=-k_{\mathrm{B}} T \int_{-\infty}^{\infty} \rho_{i, s}(E) \ln \left[1+e^{\beta(\mu-E)}\right] d E
$$

Here $\beta=1 / k_{\mathrm{B}} T$ and $\rho_{i, s}(E)=D \sum_{\zeta, n} \delta\left(E-\varepsilon_{i, s}\right)$ is the density of states (DOS) in the pristine case, where $\varepsilon_{i, s}=\zeta\left[\mu^{2}+\alpha^{2}\left(n B-\omega_{i}\right)\right]^{1 / 2}-s \mu_{\mathrm{B}} B$ are the corresponding energy levels (we omit the $\zeta$ and $n$ subscripts for simplicity), associated with the MO peaks with amplitude $A_{i}$, frequency $\omega_{i}$ and phase $s \Delta_{i}$ given by equations (3)-(5), with $\zeta= \pm 1$ for the valence band (VB) and conduction band (CB). Replacing $\rho_{i, s}(E)$, the equation (B.1) becomes $\Omega_{i}=-k_{\mathrm{B}} T D \sum_{\zeta, n} \ln \left\{1+\exp \left[\beta\left(\mu-\varepsilon_{i, s}\right)\right]\right\}$. The magnetization is given by $M_{T}=\sum_{i=1,2, s= \pm 1} M_{i, s}$, where $M_{i, s}=-\mathcal{A}^{-1}\left(\partial \Omega_{i, s} / \partial B\right)_{\mu}$. Now, under the condition 


\section{Temperature effect on the magnetic oscillations in $2 D$ materials}

$\mu>0$ and low temperatures such that $\beta \mu \gg 1$, we always have $\beta\left(\mu-\varepsilon_{i, s}\right) \gg 1$ for the $\mathrm{VB}$, so $\Omega_{i, s}^{V}(T) \simeq \Omega_{i, s}^{V}(T=0)$ and $\left(\partial \Omega_{i, s}^{V} / \partial B\right)(T) \simeq\left(\partial \Omega_{i, s}^{V} / \partial B\right)(T=0)$. On the other hand, for the $\mathrm{CB}$ we get

$$
\begin{aligned}
M_{i, s}^{C}=-\frac{1}{\mathcal{A}}\left(\frac{\partial \Omega_{i, s}^{C}}{\partial B}\right)_{\mu} & =\frac{k_{\mathrm{B}} T D}{\mathcal{A} B} \sum_{n} \ln \left[1+e^{\beta\left(\mu-\varepsilon_{i, s}\right)}\right] \\
& +\frac{D}{\mathcal{A}} \sum_{n} \frac{\partial \varepsilon_{i, s}}{\partial B} \frac{1}{1+e^{-\beta\left(\mu-\varepsilon_{i, s}\right)}}
\end{aligned}
$$

If $\mu$ is such that $\varepsilon_{i, s}(n=f) \leq \mu<\varepsilon_{i, s}(n=f+1)$, then we can write equation (B.2) as

$$
\begin{aligned}
M_{i, s}^{C} & =M_{i, s}^{C}(T=0)+\frac{k_{\mathrm{B}} T D}{\mathcal{A} B} \sum_{n=0}^{f} \ln \left[1+e^{-\beta\left(\mu-\varepsilon_{i, s}\right)}\right] \\
& +\frac{k_{\mathrm{B}} T D}{\mathcal{A} B} \sum_{n=f+1}^{\infty} \ln \left[1+e^{\beta\left(\mu-\varepsilon_{i, s}\right)}\right]+\frac{D}{\mathcal{A}} \sum_{n=0}^{f} \frac{\partial \varepsilon_{i, s}}{\partial B} \frac{1}{1+e^{\beta\left(\mu-\varepsilon_{i, s}\right)}} \\
& -\frac{D}{\mathcal{A}} \sum_{n=f+1}^{\infty} \frac{\partial \varepsilon_{i, s}}{\partial B} \frac{1}{1+e^{-\beta\left(\mu-\varepsilon_{i, s}\right)}}
\end{aligned}
$$

where $M_{i, s}^{C}(T=0)=D \sum_{n=0}^{f}\left[\left(\mu-\varepsilon_{i, s}\right) / B-\partial \varepsilon_{i, s} / \partial B\right] / \mathcal{A}$ is the CB magnetization at zero temperature. Because we are considering $\beta \mu \gg 1$, then each term in the exponential is appreciable only for $B$ such that $\varepsilon_{i, s}(B) \sim \mu$. Hence, for each term $\beta\left(\mu-\varepsilon_{i, s}\right)$, we can expand $\varepsilon_{i, s}$ around $B_{n}$, where $1 / B_{n}=n / \omega_{i}-s \Delta_{i}$ and $\varepsilon_{i, s}\left(B_{n}\right)=\mu$. Thus $\left(\mu-\varepsilon_{i, s}\right) \simeq \mu_{\mathrm{B}}\left(B_{n}-B\right) / B_{n} \Delta_{i}$. Furthermore, for the terms $\partial \varepsilon_{i, s} / \partial B$ it is good approximation to take

$$
\begin{gathered}
\frac{\partial \varepsilon_{i, s}}{\partial B}=\frac{\left(\varepsilon_{i, s}\right)^{2}-\mu^{2}+\alpha^{2} \omega_{i}}{2 B\left(\varepsilon_{i, s}+s \mu_{\mathrm{B}} B\right)} \\
\simeq \frac{\alpha^{2} \omega_{i}}{2 B \mu}=-\frac{A_{i} \mathcal{A}}{D}
\end{gathered}
$$

where $A_{i, s}$ is given by equation (3). From this we can also see that the logarithmic terms in equation (B.3) are much smaller than the exponential terms, so we can neglect them. Indeed, we always have $\ln \left\{1+\exp \left[ \pm \beta\left(\mu-\varepsilon_{i, s}\right)\right]\right\} \leq \ln 2<1$ for $n \leq f$ and $n \geq f+1$, while the ratio of amplitude between both terms is $r \equiv\left|\frac{k_{\mathrm{B}} T D}{\mathcal{A} B} / A_{i}\right|=2 k_{\mathrm{B}} T \mu / \alpha^{2} \omega_{i}$. Then, given for the $2 \mathrm{D}$ crystals we have $\alpha \sim 10 \mathrm{meV} / \sqrt{\mathrm{T}}$, and we will work with values around $\mu \sim 10^{2} \mathrm{meV}$ and $\mid s \lambda_{\mathrm{SO}}-$ nel $E_{z} \mid \sim 10 \mathrm{meV}$ so $\omega_{i} \sim 10^{2} \mathrm{~T}$, we have $r \sim 10^{-4} \mathrm{~T}[\mathrm{~K}]$. Thus, under the temperatures that we consider, is good approximation to discard the logarithms terms in equation (B.3). In this way, considering also the VB magnetization $M_{i, s}^{V}=M_{i, s}^{V}(T=0)$, and summing over $i$ and $s$, we get the total magnetization 


$$
\begin{aligned}
M_{T} & =M-\sum_{i=1,2} A_{i} \sum_{s= \pm 1}\left[\sum_{n=0}^{f} \frac{1}{1+e^{\beta \mu_{\mathrm{B}}\left(B_{n}-B\right) / B_{n} \Delta_{i}}}\right. \\
& \left.+\sum_{n=f+1}^{\infty} \frac{1}{1+e^{-\beta \mu_{\mathrm{B}}\left(B_{n}-B\right) / B_{n} \Delta_{i}}}\right],
\end{aligned}
$$

where $M$ is the magnetization at zero temperature, given by equation (2). The expression given by equation (B.5) holds under the initial assumption $\varepsilon_{i, s}(n=f) \leq$ $\mu<\varepsilon_{i, s}(n=f+1)$ for each $i, s$ peak, which in turn implies $1 / B_{i, s}(n=f) \leq 1 / B<$ $1 / B_{i, s}(n=f+1)$. Therefore, the temperature effect over the MO is to introduce factors proportional to $\left\{1+\exp \left[\beta \mu_{\mathrm{B}}\left(B_{n}-B\right) / B_{n} \Delta_{i}\right]\right\}^{-1}$ if $n \leq f f$ and proportional to $\left\{1+\exp \left[\beta \mu_{\mathrm{B}}\left(B_{n}-B\right) / B_{n} \Delta_{i}\right]\right\}^{-1}$ if $n>f$. Finally, from the properties of the arctangent and floor functions, equation $(B .5)$ can be generalized for all $B$ by introducing the exponential factors inside the arctangent in $M$, which leads to equation (7).

\section{References}

[1] Novoselov K S, Geim A K, Morozov S V, Jiang D, Katsielson M I, Grigorieva I V, Dubonos S V and Firsov A A 2005 Nature 438 197-200

[2] Geim A K and Novoselov K S 2007/Nature Materials 6 183-191

[3] Zhang Y, Tan Y W, Stormer H L and Kim P 2005 Nature 438 201-204

[4] Mas-Ballesté R, Gómez-Navarro C, Gómez-Herréro J and Zamora F 2011 Nanoscale 3 20-30

[5] Lin Z et al. 2016 2D Materials 3042001

[6] Gupta A, Sakthivel T and Seal S 2015 Progress in Materials Science 73 44-126

[7] Zhao J et al. 2016 Progress in Materials Science 83 24-151

[8] Lay G L 2015 Nature Nanotechnology 10 202-203

[9] Zhuang J, Xu X, Feng H, Li Z, Wang X and Du Y 2015 Science Bulletin 60 1551-1562

[10] Houssa M, Dimoulas A and Molle A 2015 Journal of Physics: Condensed Matter 27253002

[11] Balendhran S, Walia S, Nili H, Sriram S and Bhaskaran M 2014 Small 11 640-652

[12] Dávila M E and Lay G L 2016 Scientific Reports 6

[13] Saxena S, Chaudhary R P and Shukla S 2016 Scientific Reports 6

[14] feng Zhu F, jiong Chen W, Xu Y, lei Gao C, dan Guan D, hua Liu C, Qian D, Zhang S C and feng Jia J 2015 Nature Materials 14 1020-1025

[15] Carvalho A, Wang M, Zhu X, Rodin A S, Su H and Neto A H C 2016 Nature Reviews Materials 1

[16] Cho K, Yang J and Lu Y 2017 Journal of Materials Research 32 2839-2847

[17] Liu C C, Jiang H and Yao Y 2011 Physical Review B 84

[18] Spencer M J and Morishita T (eds) 2016 Silicene (Springer International Publishing)

[19] Neto A H C, Guinea F, Peres N M R, Novoselov K S and Geim A K 2009 Reviews of Modern Physics 81 109-162

[20] Kou L, Ma Y, Sun Z, Heine T and Chen C 2017 The Journal of Physical Chemistry Letters 8 1905-1919

[21] Tahir M and Schwingenschlgl U 2013 Scientific Reports 3

[22] Ezawa M 2015 Journal of the Physical Society of Japan 84121003

[23] Huang C, Zhou J, Wu H, Deng K, Jena P and Kan E 2016 The Journal of Physical Chemistry Letters 7 1919-1924

[24] Hsu C H et al. 2017 Physical Review B 96

[25] Wang H, Pi S T, Kim J, Wang Z, Fu H H and Wu R Q 2016 Physical Review B 94 
Temperature effect on the magnetic oscillations in $2 D$ materials

[26] Zhang H, Zhou T, Zhang J, Zhao B, Yao Y and Yang Z 2016 Physical Review B 94

[27] Ghazaryan A and Chakraborty T 2015 Physical Review B 92

[28] Ezawa M 2012 Journal of the Physical Society of Japan 81064705

[29] Liu, Luo, Xu, Tian and Ren 2017 Condensed Matter Physics 2043701

[30] Drummond N D, Zólyomi V and Fal'ko V I 2012 Physical Review B 85

[31] Du Y et al. 2014 ACS Nano 8 10019-10025

[32] Aghaei S M and Calizo I 2015 Journal of Applied Physics 118104304

[33] Yan J A, Gao S P, Stein R and Coard G 2015 Physical Review B 91

[34] Ni Z, Liu Q, Tang K, Zheng J, Zhou J, Qin R, Gao Z, Yu D and Lu J 2011 Nano Letters 12 $113-118$

[35] Abbasi A and Sardroodi J J 2018 Applied Surface Science 456 290-301

[36] Wang T, Guo W, Wen L, Liu Y, Zhang B, Sheng K and Yin Y 2017 Journal of Wuhan University of Technology-Mater. Sci. Ed. 32 213-216

[37] Sharapov S G, Gusynin V P and Beck H 2004 Physical Review B 69

[38] Tabert C J, Carbotte J P and Nicol E J 2015 Physical Review B 91

[39] Heße L and Richter K 2014 Physical Review B 90

[40] Fu Z G, Wang Z G, Li S S and Zhang P 2011 Chinese Physics B 20058103

[41] Luk'yanchuk I A 2011 Low Temperature Physics 37 45-48

[42] Uchoa B, Kotov V N, Peres N M R and Neto A H C 2008 Physical Review Letters 101

[43] Ardenghi J S, Bechthold P, Gonzalez E, Jasen P and Juan A 2015 The European Physical Journal $B 88$

[44] Ardenghi J, Bechthold P, Gonzalez E, Jasen P and Juan A 2014 Physica B: Condensed Matter $43328-36$

[45] Escudero F, Sourrouille L, Ardenghi J and Jasen P 2017 Superlattices and Microstructures 101 $537-546$

[46] Escudero F, Ardenghi J, Sourrouille L, Jasen P and Juan A 2018 Superlattices and Microstructures $113291-300$

[47] Escudero F, Ardenghi J and Jasen P 2018 Journal of Magnetism and Magnetic Materials 454 $131-138$

[48] Shoenberg D 1984 Magnetic oscillations in metals (Cambridge University Press)

[49] Escudero F, Ardenghi J, Sourrouille L and Jasen P 2017 Journal of Magnetism and Magnetic Materials 429 294-298

[50] Goerbig M O 2011 Reviews of Modern Physics 83 1193-1243

[51] Ardenghi J, Bechthold P, Jasen P, Gonzalez E and Nagel O 2013 Physica B: Condensed Matter 427 97-105

[52] Lukose V, Shankar R and Baskaran G 2007 Physical Review Letters 98

[53] Zhang S, Ma N and Zhang E 2010 Journal of Physics: Condensed Matter 22115302

[54] Escudero F, Ardenghi J S and Jasen P 2018 Journal of Physics: Condensed Matter 30275803

[55] Khalid M A, Reinders P H/P and Springford M 1988 Journal of Physics F: Metal Physics 18 1949-1964

[56] Engelsberg S and Simpson G 1970 Physical Review B 2 1657-1665

[57] Tabert C J and Carbotte J P 2014 Journal of Physics: Condensed Matter 27015008 